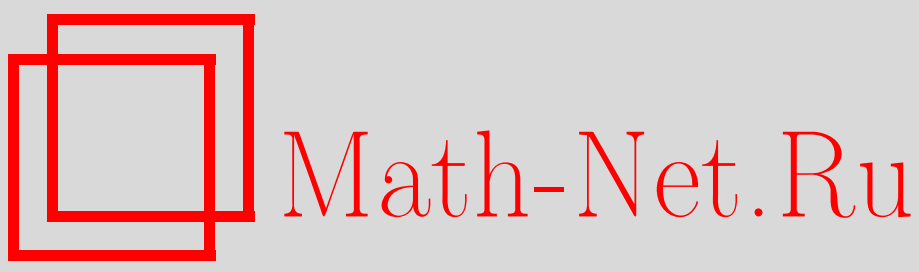

И. К. Бабенко, И. А. Тайманов, Произведения Масси в симплектических многообразиях, Матем. сб., 2000, том 191, номер 8, 3-44

DOI: https://doi.org/10.4213/sm497

Использование Общероссийского математического портала Math-Net.Ru подразумевает, что вы прочитали и согласны с пользовательским соглашением

http://www . mathnet.ru/rus/agreement

Параметры загрузки:

IP: 3.85 .7 .115

26 апреля 2023 г., $12: 46: 13$ 


\title{
Произведения Масси в симплектических многообразиях
}

\begin{abstract}
Статья посвящена изучению произведений Масси в симплектических многообразиях. Предложен общий метод, использующий симплектические раздутия, для построения симплектических многообразий, обладающих нетривиальньми произведениями Масси сколь угодно большого порядка. Найдены условия, при выполнении которых симплектическое раздутие многообразия $X$ вдоль подмногообразия $Y$ наследует нетривиальные произведения Масси с $X$ или $Y$. Как результат получена общая конструкция построения неформальных симплектических многообразий с помощью симплектических раздутий.

Библиографоия: 27 названий.
\end{abstract}

\section{§1. Введение}

В этой работе мы продолжим изучение проблемы формальности симплектических многообразий, начатое нами в [1], [2], где были построены первые примеры неформальных односвязных симплектических компактных многообразий и при этом был предложен метод построения таких многообразий с помощью симплектических раздутий.

Гладкое многообразие $X$ называется симплектическим, если на нем имеется невырожденная в каждой точке замкнутая 2-форма $\omega$. Этот объект мы будем в дальнейшем обозначать $(X, \omega)$. Первоначально возникнув в аналитической механике, симплектические многообразия давно утвердились как объект исследования чистой математики [3]. В этой работе мы будем рассматривать только замкнутые симплектические многообразия.

Естественно ожидать, что наличие симплектической структуры накладьвает сильные ограничения на топологию многообразия. Недостаток широкого спектра примеров только подтверждает эти предположения. Основньм примером компактных симплектических многообразий являются кэлеровы многообразия, и наличие кэлеровой структуры действительно накладывает сильные топологические ограничения на многообразие.

Вопрос о существовании симплектических многообразий, не имеющих кэлеровых структур, возник в начале 70-х годов, и первый пример такого многообразия был найден Тёрстоном [4], построившим 4-мерное неодносвязное симплектическое многообразие, не допускающее кэлеровой структуры. Позднее оказалось, что это многообразие - одна из комплексных, но не кэлеровых поверхностей из классификации Кодаиры. Используя этот пример, МакДафф [5] построила односвязное

Работа первого автора выполнена при поддержке Российского фонда фундаменталњных исследований (грант № 99-01-01202-а).

Работа второго автора выполнена при поддержке Российского фонда фундаментальных исследований (гранты № № 96-15-96877 и 98-01-00749).

$$
\text { (C) И.К. БАБЕнко, И.А. ТАЙмАНОв }
$$


симплектическое многообразие, не имеюшее кэлеровой структуры. Отсюда следует, что класс кэлеровых многообразий является естественным подклассом класса симплектических многообразий, но вопрос о том, насколько он узок, еще далек от полного разрешения.

Недавно Гомпф [6] показал, что любая конечно определенная группа является фундаментальной группой некоторого 4-мерного симплектического многообразия. Заметим, что у кэлерова многообразия первое число Бетти четно.

Одним из фундаментальных топологических свойств кэлеровых многообразий является их формальность, установленная в [7]. Формальность пространства $X$ означает, что его вещественный гомотопический тип полностью определяется кольцом когомологий $H^{*}(X, \mathbb{R})$.

Существование неформальных и, следовательно, некэлеровых симплектических многообразий известно сравнительно давно [8]. В частности, многообразие Кодаиры-Тёрстона, упоминавшееся вьше, неформально, хотя Тёрстоном этот факт и не обсуждался. Все примеры неформальных симплектических многообразий, известные до недавнего времени, были неодносвязными, и их неформальность проистекала из специальных свойств фундаментальной групшы. Для односвязных многообразий существовала гипотеза Лаптона-Опри [9], утверждавшая, что все односвязные симплектические многообразия формальны и в этом смысле близки к кэлеровым. В [10] был получен ряд частных результатов, подтверждающих это предположение.

Эта гипотеза была опровергнута нами в [1], [2], где был предъявлен бесконечньй набор односвязных симплектических неформальных многообразий любой четной размерности, большей 8. В частности, 10-мерное многообразие МакДафф [5] нефформально. Любое односвязное многообразие размерности не больше 6 всегда формально [11], [12]. Поэтому вопрос о сушествовании неформальных односвязных симплектических многообразий остается открытым только в размерности 8 .

Общеизвестным препятствием к формальности пространства $X$ является наличие в его когомологиях $H^{*}(X)$ высших (т.е. $n$-местных при $n \geqslant 3$ ) операций (или произведений) Масси. Именно нетривиальность тройных ординарных произведений Масси у некоторых односвязных симплектических многообразий была установлена в [2].

Независимо от проблемы формальности наличие высших произведений Масси свидетельствует о сложности топологии пространства. Высшие произведения Масси возникают в ряде вопросов гомотопической топологии, например, при описании когомологического гомоморфизма Гуревича или при описании дифференциалов спектральной последовательности Эйленберга-Мура. Одна из основных целей этой статьи - показать, что симплектические и, в частности, односвязные симплектические многообразия могут иметь нетривиальные $k$-местные матричные произведения Масси для сколь угодно больших $k$.

Работа состоит из двух частей.

В первой части мы вводим произведения Масси самого обшего вида. Частным случаем этого определения являются классические матричные произведения, введенные Мэем в 1969 г. Наш подход в определении произведений Масси основан на рассмотрении решений уравнения Маурера-Картана на алгебре матричных дифференциальных форм данного многообразия

$$
d A-\bar{A} \cdot A \equiv 0 \bmod K(A)
$$


Здесь $A$ - верхнетреугольная матрица, элементами которой являются дифференциальные формы на исходном многообразии, $K(A)$ - некоторый подмодуль, ассоциированный с данной матрицей.

То, что определяюшая система любого матричного произведения Масси может быть расположена в матрицу, удовлетворяющую уравнению Маурера-Картана типа (1), было замечено еше Мэем, но этот подход не получил никакого развития.

Решение $A$ уравнения (1) можно трактовать как формальную связность. Тогда соответствуюшие обобщенные произведения Масси будут классами когомологий “формы кривизны”

$$
\mu(A)=d A-\bar{A} \cdot A
$$

связности $A$. Этот дифференциально-геометрический подход к определению произведений Масси позволяет достаточно просто устанавливать некоторые основные свойства этих произведений.

В первой части мы кратко рассматриваем связь произведений Масси с гомоморфизмами Гуревича и надстройки, а также со спектральной последовательностью Эйленберга-Мура.

Во второй части настояшей работы рассматривается применение теории произведений Масси к симплектическим многообразиям. Для построения новых симплектических многообразий из уже имеющихся используется три основных приема: симплектические расслоения, расслоенные связные суммы и симплектические раздутия. Хотя при построении симплектических многообразий с нетривиальными произведениями Масси можно применять конструкцию симплектического расслоения (например, многообразие Кодаиры-Тёрстона), наиболее перспективной здесь представляется конструкция симплектического раздутия [13], [5].

Пусть $(X, \omega)$ - симплектическое многообразие и $Y \subset X-$ симплектическое подмногообразие. Обозначим через $\widetilde{X}$ симплектическое раздутие $X$ вдоль $Y$. Главньй вопрос, изучаемьй в этой части, - что может происходить с высшими операциями Масси при симплектическом раздутии? Он распадается на следующие две части.

1) "Выживание". Пусть $X$ обладает нередуцируемыми высшиими (матричными) произведениями Масси. При каких условиях на $Y$ эти операции будут выживать в $\widetilde{X}$ ?

2) "Наследование". Предположим, что $Y$ обладает нетривиальными высшими (матричными) произведениями Масси. При каких условиях на $X$ эти операции будут наследоваться $\widetilde{X}$ ?

Сформулируем основные результаты в этом направлении.

Теорема А. Пусть односвязное симплектическое многообразие $X$ имеет нередуцируемое обобщенное произведение Масси размерности $k$. Тогда для любого симплектического подмногообразия $Y \subset X$ такого, что $\operatorname{codim} Y>k$, соответствующее симплектическое раздутие $\widetilde{X}$ такэе имеет нередуцируемое обобщенное произведение Масси размерности $k$.

Теорема В. Пусть симплектическое многообразие $(Y, \omega)$ имеет нетривиальное матричное п-местное произведение Масси $\left\langle S_{1}, \ldots, S_{n}\right\rangle$, әде $S_{i} \in$ $N\left(H^{1}(Y)\right), 1 \leqslant i \leqslant n$. Тогда для любого симплектического вложсения $Y \subset X$ коразмерности не меньше $2(n+1)$ соответствующее симплектическое раздутие $\widetilde{X}$ имеет нетривиальное $п$-местное произведение Масси $\left\langle\widetilde{S}_{1}, \ldots, \widetilde{S}_{n}\right\rangle$, əде $\widetilde{S}_{i} \in N\left(H^{3}(\widetilde{X})\right), 1 \leqslant i \leqslant n$. 
ТеОрема С. Пусть симплектическое многообразие $(Y, \omega)$ имеет нетривиальное тройное матричное произведение Масси. Тогда для любого симплектического вложения $Y \subset X$ коразмерности не меньие 8 соответствующее симплектическое раздутие $\widetilde{X}$ такжсе имеет нетривиальное тройное матричное произведение Масси.

Частный случай теоремы С был установлен нами в [1], [2], причем достаточно общие рассуждения, примененные к раздутиям $\mathbb{C} P^{n}$ вдоль вложенных многообразий Кодаиры-Тёрстона и изложенные в [1], дают оценку 8 на коразмерность. Позднее мы указали в [2], что для данного конкретного многообразия оценка на коразмерность может быть снижена до 6.

В [2] мы поставили проблему о наследовании нетривиальных произведений Масси в обшем случае. Она обсуждалась в [14], где была доказана теорема С для случая ординарных произведений Масси.

ТЕОрема D. Пусть симплектическое многообразие $(Y, \omega)$ имеет сильно нередуиируемое четверное матричное произведение Масси $\left\langle S_{1}, S_{2}, S_{3}, S_{4}\right\rangle$. Тогда для любого симплектического вложения $Y \subset X$ такого, что

$$
\operatorname{codim} Y>2 \operatorname{sdeg}\left\langle S_{1}, S_{2}, S_{3}, S_{4}\right\rangle
$$

соответствующее симплектическое раздутие $\widetilde{X}$ обладает нетривиальным четверным матричным произведением Масси.

Теоремы A, B, С и D дают возможность строить новые многообразия, обладающие нетривиальными произведениями Масси, совершая симплектическое раздутие $X$ вдоль подмногообразия $Y$. При этом необходимо, чтобы либо $X$, либо $Y$ имели нетривиальные произведения Масси. Поэтому чтобы эта схема работала, надо иметь “стартовый набор” симплектических многообразий с нетривиальными произведениями Масси. Существование такого набора гарантирует теорема 6 , которую мы докажем в $\S 3$ :

Для любого натурального $k$ существуют симплектические многообразия, обладающие матричными произведениями Масси веса строго $2 k$.

Примеры таких многообразий построены в предложении 8.

Эти результаты показывают, что сушествование симплектической структуры не накладывает сильных ограничений на гомотопический тип многообразия. Мы хотим высказать следующую гипотезу.

ГИПотеЗА. Для каждого конечного полиәдра $P$ и каждого $N$ существуют симплектическое многообразие $X$ и вложение $f: P \rightarrow X$ такие, что

$$
f_{*}: \pi_{k}(P) \rightarrow \pi_{k}(X)-\text { мономорфизм } п р и k \leqslant N
$$

Для $N=1$ и $\operatorname{dim} P=2$ эта гипотеза следует из результатов Гомпфа [6]. Авторы благодарят Д. ван Стратена и А. Тралле за полезные обсуждения. 


\section{§ 2. Произведения Масси и некоторые конструкции теории гомотопий}

2.1. Алгебра форм и ее минимальная модель. Пусть $\mathscr{A}$ - дифференциальная градуированная алгебра над полем $k$. Это означает, что $\mathscr{A}$ представляется в виде прямой суммы подпространств $\mathscr{A}^{l}$, образованных однородными элементами степени $l \geqslant 0$ :

$$
\mathscr{A}=\bigoplus_{l \geqslant 0} \mathscr{A}^{l}, \operatorname{deg} a=l \text { при } a \in \mathscr{A}^{l},
$$

и на $\mathscr{A}$ заданы следующие линейные операции: ассоциативное умножение

$$
\wedge: \mathscr{A}^{l} \times \mathscr{A}^{m} \rightarrow \mathscr{A}^{l+m}, \quad l, m \geqslant 0,
$$

и дифференциал

$$
d: \mathscr{A}^{l} \rightarrow \mathscr{A}^{l+1}, \quad l \geqslant 0
$$

При этом вьполняются следующие условия:

1) $a \wedge b=(-1)^{l m} b \wedge a$ при $a \in \mathscr{A}^{l}, b \in \mathscr{A}^{m}$;

2) $d(a \wedge b)=d a \wedge b+(-1)^{l} a \wedge d b$ при $a \in \mathscr{A}^{l}$ (правило Лейбница);

3) $d^{2}=0$.

Гомоморфизмом дифференциальных градуированных алгебр $\left(\mathscr{A}, d_{\mathscr{A}}\right)$ и $\left(\mathscr{B}, d_{B}\right)$ над полем $k$ называется $k$-линейное отображение

$$
f: \mathscr{A} \rightarrow \mathscr{B},
$$

сохраняющее градуировку:

$$
f\left(\mathscr{A}^{l}\right) \subset \mathscr{B}^{l} \text { при } l \geqslant 0,
$$

и удовлетворяюшее условиям

$$
\begin{gathered}
f(a \wedge b)=f(a) \wedge f(b), \quad a, b \in \mathscr{A}, \\
d_{\mathscr{B}} f(a)=f\left(d_{\mathscr{A}} a\right), \quad a \in \mathscr{A} .
\end{gathered}
$$

Когомологии алгебры $\left(\mathscr{A}, d_{\mathscr{A}}\right)$ определяются естественным образом:

$$
H^{l}\left(\mathscr{A}, d_{\mathscr{A}}\right)=\operatorname{Ker}\left(d_{\mathscr{A}}: \mathscr{A}^{l} \rightarrow \mathscr{A}^{l+1}\right) / \operatorname{Im}\left(d_{\mathscr{A}}: \mathscr{A}^{l-1} \rightarrow \mathscr{A}^{l}\right) .
$$

При этом каждому замкнутому элементу (или коциклу) $a \in \mathscr{A}$, т.е. такому, что $d_{\mathscr{A}} a=0$, отвечает его класс когомологий $[a] \in H^{+}\left(\mathscr{A}, d_{\mathscr{A}}\right)$. Если $[a]=0$, то элемент $a$ называется точным.

Гомоморфизм $f:\left(\mathscr{A}, d_{\mathscr{A}}\right) \rightarrow\left(\mathscr{B}, d_{\mathscr{B}}\right)$ переводит замкнутые элементы в замкнутые, а точные - в точные. Поэтому он индуцирует гомоморфизм

$$
f^{*}: H^{*}\left(\mathscr{A}, d_{\mathscr{A}}\right) \rightarrow H^{*}\left(\mathscr{B}, d_{\mathscr{B}}\right)
$$

по формуле $f^{*}[a]=[f(a)]$.

Алгебра $(\mathscr{A}, d)$ называется связной, если $H^{0}(\mathscr{A}, d)=k$. Связная алгебра $\mathscr{A}$ называется односвязной, если $H^{1}(\mathscr{A}, d)=0$. Если не оговорено противное, то мы будем считать, что алгебра $\mathscr{A}$ односвязна. 
Мы также будем считать, что $\mathscr{A}$ аугментирована, т.е. задан эпиморфизм дифференциальных градуированных алгебр

$$
\varepsilon: \mathscr{A} \rightarrow k,
$$

где алгебра $k$ образована элементами нулевой степени и снабжена нулевым дифференциалом. Идеал $I=\operatorname{Ker} \varepsilon$ называется идеалом аугментации.

Основным примером в этой работе будет алгебра $\mathscr{E} *(X)$ гладких внешних форм на связном гладком многообразии $X$. Если $X$ односвязно, то $\mathscr{E} *(X)$ - (когомологически) односвязная алгебра.

Пусть $i: x \rightarrow X$ - вложение точки $x \in X$ в $X$. Индуцированное отображение задает аугментацию

$$
\varepsilon=i^{*}: \mathscr{E}^{*}(X) \rightarrow \mathscr{E}^{*}(x)=k=\mathbb{R} .
$$

Идеал аугментации $I$ состоит из всех форм положительной степени и из всех гладких функций $\varphi: X \rightarrow \mathbb{R}$ таких, что $\varphi(x)=0$.

$\mathrm{B}$ дальнейшем мы для простоты будем всюду считать, что $k=\mathbb{R}$.

Дифференциальная градуированная алгебра $\left(\mathscr{M}, d_{\mathscr{M}}\right)$ называется минимальной, если

1) $\mathscr{M}^{0}=\mathbb{R}, d\left(\mathscr{M}^{0}\right)=0$ и умножение на элементы из $\mathscr{M}^{0}$ совпадает с умножением на элементы из основного поля $k=\mathbb{R}$;

2) $\mathscr{M}^{+}=\bigoplus_{l>0} \mathscr{M}^{l}$ свободно порождена однородньми образуюшими $x_{1}, \ldots$, $x_{n}, \ldots$ :

$$
\mathscr{M}^{+}=\wedge\left(x_{1}, \ldots\right),
$$

при этом для каждого $l>0$ существует конечное число таких образуюших степени $l$ и

$$
\operatorname{deg} x_{i} \leqslant \operatorname{deg} x_{j} \text { при } i \leqslant j ;
$$

3) дифференциал $d$ разложим:

$$
d x_{i} \in \wedge\left(x_{1}, \ldots, x_{i-1}\right) \text { при } i \geqslant 1,
$$

т.е. $d x_{i}$ выражается как полином от $x_{1}, \ldots, x_{i-1}$.

Очевидно, что минимальная алгебра $\mathscr{M}$ односвязна тогда и только тогда, когда $\mathscr{M}^{1}=0$. При этом $\operatorname{deg} x_{i} \geqslant 2$ при $i \geqslant 1$ и условие разложимости переписывается как

$$
d\left(\mathscr{M}^{+}\right) \subset \mathscr{M}^{+} \wedge \mathscr{M}^{+},
$$

где $\mathscr{M}^{+} \wedge \mathscr{M}^{+}$- линейное подпространство, порожденное разложимыми элементами.

Алгебра $\left(\mathscr{M}, d_{\mathscr{M}}\right)$ назьвается минимальной моделью алгебры $\left(\mathscr{A}, d_{\mathscr{A}}\right)$, если

1) алгебра $\left(\mathscr{M}, d_{\mathscr{M}}\right)$ минимальна;

2) сушествует гомоморфизм $h:\left(\mathscr{M}, d_{\mathscr{M}}\right) \rightarrow\left(\mathscr{A}, d_{\mathscr{A}}\right)$, индуцирующий изоморфизм

$$
h^{*}: H^{*}\left(\mathscr{M}, d_{\mathscr{M}}\right) \rightarrow H^{*}\left(\mathscr{A}, d_{\mathscr{A}}\right) .
$$

В дальнейшем для гомоморфизмов, удовлетворяющих этому условию, будет использоваться термин квазиизоморфизм.

Фундаментальная теорема Сулливана [15], [7] гласит, что 
Теорема 1 (Сулливан). Если $\left(\mathscr{A}, d_{\mathscr{A}}\right)$ - односвязная дифференциальная градуированная алгебра такая, что $\operatorname{dim} H^{l}(\mathscr{A})<\infty$ для кажсдого $l \geqslant 0$, то существует единственная ( с точностью до изоморфизма) минимальная модель алгебры $\mathscr{A}$.

Примером такой алгебры является алгебра гладких форм на односвязном компактном многообразии.

Если $\mathscr{A}=\mathscr{E} *(X)$, то минимальная модель $\mathscr{M}_{X}$ алгебры $\mathscr{A}$ называется также минимальной (вещественной) моделью пространства $X$. Так как по теореме де Рама когомологии алгебры гладких форм на $X$ изоморфны вещественным когомологиям $X$, то гомоморфизм

$$
h:\left(\mathscr{M}_{X}, d_{\mathscr{M}}\right) \rightarrow\left(\mathscr{E}^{*}(X), d_{X}\right)
$$

порождает изоморфизм

$$
h^{*}: H^{*}\left(\mathscr{M}_{X}\right) \rightarrow H^{*}(X)
$$

где $H^{*}(X)=H^{*}(X, \mathbb{R})$.

Примеры. 1) Если $X=S^{2 n+1}, n \geqslant 1$, то $\mathscr{M}_{X}^{+}$порождена элементом $x$ с $d x=0$ и $\operatorname{deg} x=2 n+1$.

2) Если $X=S^{2 n}, n \geqslant 1$, то $\mathscr{M}_{X}^{+}$порождена элементами $x$ и $y$, где $\operatorname{deg} x=2 n$, $\operatorname{deg} y=4 n-1, d x=0, d y=x^{2}$.

Важное свойство минимальных моделей содержится в следующем утверждении $[15],[7]:$

ТЕОремА 2 (Сулливан). Каждое гладкое отображение

$$
f: X \rightarrow Y
$$

индуцирует гомоморфизм минимальных моделей

$$
\widehat{f}: \mathscr{M}_{Y} \rightarrow \mathscr{M}_{X}
$$

такой, что диаграмма

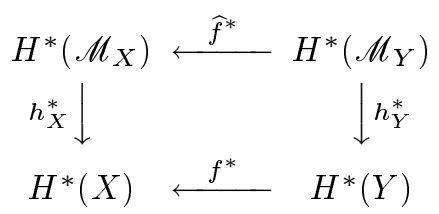

коммутативна.

Другие важные свойства минимальных моделей указаны в [15], [7], и мы отметим лишь одно из них: имеется естественный изоморфизм

$$
\operatorname{Hom}\left(\pi_{l}(X), \mathbb{R}\right)=\mathscr{M}_{X}^{l} / \mathscr{M}_{X}^{+} \wedge \mathscr{M}_{X}^{+}
$$

т.е. неразложимые элементы из $\mathscr{M}_{X}^{l}$ находятся во взаимно однозначном соответствии с гомоморфизмами из $\pi_{l}(X)$ в $\mathbb{R}$. Заметим, что мы подразумеваем здесь, что для $X$ сушествует минимальная модель, а она строится лишь для односвязных и нильпотентных неодносвязных пространств. 
В неодносвязном случае мы ограничимся описанием минимальной модели для нильмногообразий, т.е. факторпространств нильпотентных групп $G$ по равномерным решеткам $\Gamma \subset G$.

Пусть $G$ - односвязная нильпотентная группа Ли, $\mathscr{G}$ - ее алгебра Ли и $\mathscr{G}^{*}-$ двойственная ей алгебра, т.е. алгебра линейных функций $f: \mathscr{G} \rightarrow \mathbb{R}$.

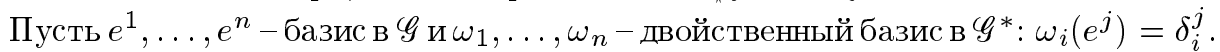
Структурные константы $c_{k}^{i j}$ определяются по формуле

$$
\left[e^{i}, e^{j}\right]=\sum_{k=1}^{n} c_{k}^{i j} e^{k}
$$

и по теореме Мальцева [16] группа $G$ содержит дискретную подгруппу $\Gamma$ с компактным факторпространством $G / \Gamma$ тогда и только тогда, когда в подходящем базисе $e^{1}, \ldots, e^{n}$ все структурные константы рациональны.

Группа $G$ диффеоморфна $\mathbb{R}^{n}$ и ее элементы можно отождествить с векторами $\xi=\sum_{i} \xi_{i} e^{i} \in \mathbb{R}^{n}$. Умножение задается формулой Кемпбелла-Хаусдорфа

$$
\left(\sum_{i} \xi_{i} e^{i}\right) \times\left(\sum_{j} \eta_{j} e^{j}\right)=\sum_{k} P_{k}\left(\xi_{1}, \ldots, \xi_{n}, \eta_{1}, \ldots, \eta_{n}\right) e^{k}
$$

где $P_{1}, \ldots, P_{n}$ - многочлены (это следует из нильпотентности алгебры $\mathscr{G}$ ). Если $c_{k}^{i j}$ рациональны, то $P_{1}, \ldots, P_{n}$ - многочлены с рациональньми коэффициентами и подгруппа, порожденная векторами $e^{1}, \ldots, e^{n}$, является (равномерной) решеткой, т.е. дискретной подгруппой $\Gamma$ с компактным факторпространством $G / \Gamma$.

Пусть $\mathscr{M}_{X}$ - минимальная модель $X=G / \Gamma$, где $\Gamma$ - произвольная решетка в нильпотентной групше $G$ с компактньг факторпространством $G / \Gamma$. Тогда $\mathscr{M}_{X}^{+}$ порождена элементами $a_{1}, \ldots, a_{n}$, где

$$
\begin{gathered}
\operatorname{deg} a_{1}=\cdots=\operatorname{deg} a_{n}=1, \\
d a_{k}=\sum_{i, j} c_{k}^{i j} a_{i} \wedge a_{j}
\end{gathered}
$$

Отображение $\mathscr{M}_{X} \rightarrow \mathscr{E} *(X)$ имеет вид

$$
a_{k} \rightarrow \omega_{k}
$$

где $\omega_{k}$ - левоинвариантная 1-форма на $X$, порожденная одноименным элементом из $\mathscr{G}^{*}$. По теореме Номидзу [17] это отображение индуцирует изоморфизм

$$
H^{*}\left(\mathscr{M}_{X}\right) \simeq H^{*}(X)
$$

и минимальная модель нильмногообразия совпадает с комплексом, порожденным левоинвариантньми 1-формами.

Заметим, что для нильмногообразий минимальные модели неодносвязны. 
ПРИМЕР. Пусть $G$ - группа матриц вида

$$
\left(\begin{array}{lll}
1 & x & z \\
0 & 1 & y \\
0 & 0 & 1
\end{array}\right), \quad x, y, z \in \mathbb{R}
$$

а $\Gamma=G_{\mathbb{Z}}-$ подгруппа, содержашая все элементы с $x, y, z \in \mathbb{Z}$. Базис левоинвариантных 1-форм на $G$ может быть выбран в виде

$$
\omega_{1}=d x, \quad \omega_{2}=d y, \quad \omega_{3}=x d y-d z
$$

Минимальная модель $\mathscr{M}_{X}$ нильмногообразия Гейзенберга $X=G / G_{\mathbb{Z}}$ порождена элементами $a_{1}, a_{2}, a_{3}$ степени 1 , где

$$
d a_{1}=d a_{2}=0, \quad d a_{3}=a_{1} \wedge a_{2}
$$

2.2. Формальность. Каждой минимальной алгебре $(\mathscr{M}, d)$ отвечает алгебра ее когомологий $\mathscr{H}^{*}(\mathscr{M})$, которую мы будем рассматривать как дифференциальную градуированную алгебру с нулевым дифференциалом: $\left(\mathscr{H}^{*}(\mathscr{M}), 0\right)$.

Если сушествует гомоморфизм

$$
f:(\mathscr{M}, d) \rightarrow\left(\mathscr{H}^{*}(\mathscr{M}), 0\right)
$$

индуцирующий изоморфизм когомологий

$$
f^{*}: \mathscr{H}^{*}(\mathscr{M}) \simeq \mathscr{H}^{*}(\mathscr{M})
$$

то алгебра $\mathscr{M}$ называется формальной.

Сушествование такого изоморфизма означает, что алгебра $(\mathscr{M}, d)$ является минимальной моделью для своего кольца когомологий, снабженного нулевым дифференциалом. Построение минимальной модели для произвольной алгебры $\left(\mathscr{A}, d_{\mathscr{A}}\right)$ можно провести индуктивно, и поэтому формальность означает, что по кольцу когомологий $\mathscr{H}^{*}(\mathscr{M})$ можно полностью восстановить минимальную алгебру $\mathscr{M}$.

Дифференциальная градуированная алгебра $\left(\mathscr{A}, d_{\mathscr{A}}\right)$ называется формальной, если ее минимальна модель $\mathscr{M}(\mathscr{A})$ формальна. В этом случае по кольцу когомологий $\mathscr{H}^{*}\left(\mathscr{A}, d_{\mathscr{A}}\right)=\mathscr{H}^{*}\left(\mathscr{M}(\mathscr{A}), d_{\mathscr{M}}\right)$ восстанавливается минимальная модель алгебры $\left(\mathscr{A}, d_{\mathscr{A}}\right)$.

В случае, когда алгебра гладких форм $\mathscr{E} *(X)$ на гладком многообразии $X$ формальна, то само многообразие $X$ называется формальньм.

Понятие формальности обобшается на компактные односвязные или нильпотентные полиэдры. При этом алгебра $\mathscr{E} *(X)$ заменяется на алгебру кусочно-полиномиальных форм [15], [18].

Примером формальных пространств являются компактные симметрические пространства и компактные кэлеровы многообразия [7], а также односвязные компактные многообразия (без края) размерности $\leqslant 6$ [11], [12].

Примером неформальной алгебры является указанная в п. 2.1. минимальная модель трехмерного нильмногообразия Гейзенберга. Действительно, если существует гомоморфизм

$$
f:\left(\mathscr{M}_{X}, d\right) \rightarrow\left(H^{*}(X), 0\right)
$$


индуцирующий изоморфизм в когомологиях, то

$$
f\left(a_{1}\right) \neq 0, \quad f\left(a_{3}\right)=0
$$

и, следовательно, $f\left(a_{1} \wedge a_{3}\right)=0$, но элемент $a_{1} \wedge a_{3}$ реализует ненулевой класс когомологий.

Критерий формальности минимальной алгебры был указан в [7]. Чтобы сформулировать его, заметим, что минимальная алгебра представляется в виде тензорного произведения внешних алгебр $\Lambda\left(V_{l}\right)$, где $V_{l}$ - пространства, порожденные $l$-мерньми образующими:

$$
\mathscr{M}=\bigotimes_{l \geqslant 0} \Lambda\left(V_{l}\right)
$$

В каждом $V_{l}$ выделим подпространство $C_{l}$, образованное замкнутыми элементами.

Теорема 3 [7]. Минимальная алгебра $(\mathscr{M}, d)$ формальна, если и только если в каждом пространстве $V_{l}$ существует дополнение $N_{l} \kappa C_{l}$ :

$$
V_{l}=C_{l} \oplus N_{l}
$$

такое, что каждый замкнутый әлемент из идеала $I_{N}=I\left(\oplus N_{l}\right)$, порожденного әлементами из $N_{1}, N_{2}, \ldots$, является точным.

Использовать этот критерий не всегда бывает удобно, поэтому часто используют следуюший тест: найти в $H^{*}(\mathscr{M}, d)$ ненулевые элементы, представимые в виде произведений Масси. Если нетривиальные произведения Масси есть, то алгебра $(\mathscr{M}, d)$ неформальна. Обратное, как показывает пример из [19], неверно. Мы обсудим связь формальности с произведениями Масси подробнее после того, как определим эти произведения.

2.3. Уравнения Маурера-Картана в дифференциальных алгебрах и обобщенные произведения Масси. Пусть $(\mathscr{A}, d)$ - дифференциальная градуированная алгебра над $k(=\mathbb{R})$ с аугментацией. Пусть $M(\mathscr{A})$ - множество всевозможных верхнетреугольных полубесконечных матриц с элементами из $\mathscr{A}$ и нулями по диагонали, причем число ненулевых элементов конечно.

Таким образом, для каждой матрицы

$$
A=\left(a_{i j}\right)_{i, j \geqslant 1} \in M(\mathscr{A})
$$

имеет место

$$
a_{i j} \in \mathscr{A}, \quad a_{i j}=0 \text { при } j \leqslant i \text { и } i, j \geqslant n+1
$$

для некоторого $n$. Заметим, что условие

$$
a_{i j}=0 \text { при } i, j \geqslant n+1
$$

выделяет в $M(\mathscr{A})$ подмножество $M_{n}(\mathscr{A})$, состоящее из $(n \times n)$-матрищ с элементами из $\mathscr{A}$. Сушествует естественная фильтрация

$$
M_{1}(\mathscr{A}) \subset M_{2}(\mathscr{A}) \subset \cdots \subset M_{n}(\mathscr{A}) \subset \cdots \subset M(\mathscr{A}) .
$$

На $M(\mathscr{A})$ заданы операции сложения и умножения, преврашающие $M(\mathscr{A})$ в алгебру над $k=\mathbb{R}$. При этом $M_{n}(\mathscr{A}), n \geqslant 1$, являются подалгебрами $M(\mathscr{A})$. 
Зададим на $M(\mathscr{A})$ дифференциал $d$ по формуле

$$
d A=\left(d a_{i j}\right)_{i, j \geqslant 1}
$$

Если $a \in \mathscr{A}^{k}$ - однородный элемент степени $k$, то отображение

$$
a \rightarrow \bar{a}=(-1)^{k} a
$$

порождает автоморфизм порядка 2 (инволюцию) алгебры $\mathscr{A}$. Этот автоморфизм продолжается на $M(\mathscr{A})$ по правилу $\bar{A}=\left(\bar{a}_{i j}\right)_{i, j \geqslant 1}$, и дифференциал $(2)$ удовлетворяет обобшенному правилу Лейбница

$$
d(A B)=(d A) B+\bar{A}(d B)
$$

Алгебра $M$ имеет естественную биградуировку:

$$
M=\sum_{p \geqslant 1, k \geqslant 0} M^{p, k} .
$$

Действительно, пусть $(a)_{i j}$ означает матрицу, у которой все элементы равны нулю, кроме элемента, стоящего в $i$-й строке и $j$-м столбце и равного $a \in \mathscr{A}$. Теперь определим $M^{p, k}$ как подпространство $M$, порожденное элементами вида

$$
(a)_{i,(i+p)}, \quad \text { где } a \in \mathscr{A}^{k}, \quad i=1,2, \ldots
$$

По отношению к операции умножения мы получаем отображение

$$
M^{p, k} \otimes M^{q, l} \stackrel{\cdot}{\longrightarrow} M^{p+q, k+l},
$$

которое не удовлетворяет условиям градуированной коммутативности.

Еще одной структурой на $M$ является биградуированная скобка Ли

$$
M^{p, k} \otimes M^{q, l} \stackrel{[\cdot, \cdot]}{\longrightarrow} M^{p+q, k+l},
$$

которая на однородных элементах задается равенством

$$
[A, B]=A \cdot B-(-1)^{k l} B \cdot A, \quad A \in M^{p, k}, \quad B \in M^{q, l}
$$

Непосредственно проверяется, что эта скобка превращает $M$ в дифференциальную супералгебру Ли со стандартными свойствами:

1) $[A, B]=-(-1)^{k l}[B, A]$;

2) $d[A, B]=[d A, B]+[\bar{A}, d B]$;

3) $(-1)^{k m}[[A, B], C]+(-1)^{l k}[[B, C], A]+(-1)^{l m}[[C, A], B]=0$ для любых $A \in M^{p, k}, B \in M^{q, l}, C \in M^{r, m}$. 
Теперь для каждой (не обязательно однородной) матрицы $A \in M$ определим ее ядро $\operatorname{Ker} A$ как некоторый двусторонний $\mathscr{A}$-подмодуль в $M$. Положим по определению, что $\operatorname{Ker} A$ как $\mathscr{A}$-подмодуль порожден матрицами $(1)_{i j}$ такими, что $A \cdot(1)_{i j}=(1)_{i j} \cdot A=0$. Непосредственно из определения получаем, что

$$
A B=B A=0
$$

для любой матрицы $B \in \operatorname{Ker} A$.

Заметим, что $\operatorname{Ker} A$ в обшем случае не является идеалом в $M$ ни по отношению к умножению ', ни по отношению к скобке Ли $[\cdot, \cdot]$. Тем не менее, в $\operatorname{Ker} A$ имеется подмодуль $\operatorname{Ker}^{\prime} A$, являющийся двусторонним идеалом как в смысле умножения, так и в смысле скобки $[\cdot, \cdot]$. Опишем его. Пусть $m$ - номер первого ненулевого столбца в $A, k$ - номер последней ненулевой строки. Определим $\operatorname{Ker}^{\prime} A$ как $\mathscr{A}$-подмодуль $M$, порожденный матрицами $(1)_{i j}$, где $i<m, j>k$.

Далее введем в рассмотрение оператор Маурера-Картана $\mu: M \rightarrow M$, положив

$$
\mu(A)=d A-\bar{A} \cdot A .
$$

ОПРеДЕЛЕНИЕ 1. Матрица $A \in M$ называется матрицей формальной связности, если она удовлетворяет уравнению Маурера-Картана на алгебре $\mathscr{A}$

$$
d A-\bar{A} \cdot A \equiv 0 \bmod \operatorname{Ker} A .
$$

Иначе говоря, $A$ - формальная связность, если $\mu(A) \in \operatorname{Ker} A$.

ОПРЕДЕЛЕНИЕ 2. Если $A$ - некоторая формальная связность, то матрица $\mu(A)$ называется матрицей кривизнь формальной связности.

Пример. Пусть $A$ - верхнетреугольная $(n+1) \times(n+1)$-матрица с нулями по диагонали вида

$$
A=\left(\begin{array}{cccccc}
0 & a_{1} & * & \ldots & * & * \\
0 & 0 & a_{2} & \ldots & * & * \\
\ldots & \ldots & \ldots & \ldots & \ldots & \ldots \\
0 & 0 & 0 & \ldots & 0 & a_{n} \\
0 & 0 & 0 & \ldots & 0 & 0
\end{array}\right),
$$

где $a_{1}, \ldots, a_{n}$ не равны нулю. Тогда $\operatorname{Ker} A \cap M_{n+1}(\mathscr{A})$ порождено над полем $k$ единственной матрищей с единичньм элементом в пересечении $(n+1)$-й строки и $(n+1)$-й диагонали.

Условие (3) при этом принимает вид

$$
d A-\bar{A} \cdot A=\left(\begin{array}{cccc}
0 & \ldots & 0 & \tau \\
0 & \ldots & 0 & 0 \\
\ldots \ldots & \ldots & \ldots \\
0 & \ldots & 0 & 0
\end{array}\right) .
$$

ЛЕмма 1 (тождество Бианки). Для любой матрицы $A \in M$ справедливо тождество

$$
d \mu(A)=\overline{\mu(A)} \cdot A-A \cdot \mu(A) .
$$


ДокАЗАТЕЛьство. Из определения оператора $\mu$ имеем

$$
\begin{aligned}
d \mu(A) & =-d(\bar{A} \cdot A)=-d \bar{A} \cdot A-A \cdot d A=\overline{d A} \cdot A-A \cdot d A \\
& =\overline{(\mu(A)+\bar{A} \cdot A)} \cdot A-A(\mu(A)+\bar{A} \cdot A) \\
& =\overline{\mu(A)} \cdot A+A \cdot \bar{A} \cdot A-A \cdot \mu A-A \cdot \bar{A} \cdot A \\
& =\overline{\mu(A)} \cdot A-A \cdot \mu(A) .
\end{aligned}
$$

Лемма доказана.

СлЕДСТВИЕ 1. Матрица кривизны формальной связности замкнута.

ДоказАтЕльство. Если $A$ - матрица формальной связности, то $\mu(A) \in \operatorname{Ker} A$, следовательно, и $\overline{\mu(A)} \in \operatorname{Ker} A$. Из тождества Бианки немедленно получаем, что $d \mu(A)=0$.

Далее для каждого замкнутого элемента $a \in \mathscr{A}, d a=0$, соответствующий класс когомологий будем обозначать через $[a] \in H^{*}(\mathscr{A})$. Если $A \in M(\mathscr{A}), d A=0$, - замкнутая матрица, то через $[A]=\left(\left[a_{i j}\right]\right)_{i, j \geqslant 1} \in M\left(H^{*}(\mathscr{A})\right)$ будем обозначать соответствующую матрицу из классов когомологий матричных элементов $a_{i j}$.

Следствие 1 гарантирует корректность следуюшего определения.

ОПредЕлЕниЕ $3^{1}$. Пусть $A$-произвольное решение уравнения Маурера-Картана, тогда матричные элементы матрицы $[\mu(A)]$ называются обобщенными произведениями Масси.

Иньми словами, обобщенные произведения Масси - это классы когомологий матриц кривизны формальных связностей. Говоря языком дифференциальной геометрии, обобшенные произведения Масси измеряют отличие формальных связностей от плоских. Если все обобщенные произведения Масси тривиальны, то все формальные связности плоские (см. п. 2.4).

ЗАмечАния. 1) Классические $n$-местные произведения Масси (см. п. 2.3) будут отличаться знаком от соответствующих обобщенных произведений Масси. Мы специально прибегли к замене знака в определении 1, чтобы подчеркнуть параллель конструкций абстрактной гомологической алгебры и дифференциальной геометрии.

2) Можно показать, что если $\alpha \in H^{*}(\mathscr{A})$ является обобщенным произведением Масси, то $t \alpha, t \in k$, также является произведением Масси. В силу равенства $[\mu(A)]=[d A-\bar{A} \cdot A]=-[\bar{A} \cdot A]$ мы получаем, что обобщенные произведения Масси - это матричные элементы матрицы $[\bar{A} \cdot A]$.

Из двух равнозначных терминов “решение уравнения Маурера-Картана" и “формальная связность” условимся в дальнейшем употреблять только первьй.

Пусть $\varepsilon: \mathscr{A} \rightarrow k$ - аугментация и $I=\operatorname{Ker} \varepsilon-$ аугментированный идеал в $\mathscr{A}$. Если $\mathscr{A}$ связна, т.е. $\mathscr{A}^{0}=k$, то $I=\mathscr{A}^{+}$- идеал элементов положительной степени;

\footnotetext{
${ }^{1}$ Заметим, что произведения Масси определяются в более общей ситуации: когда $\mathscr{A}$ является алгеброй над коммутативным кольцом (например, $\mathbb{Z}$ ) и умножение в $\mathscr{A}$ не обязательно косокоммутативно $\left(a \wedge b=(-1)^{p q} b \wedge a\right)$, а лишь ассоциативно. Таковы, например, классические произведения Масси в $H^{*}(X, \mathbb{Z})$.
} 
эта ситуация имеет место, если $\mathscr{A}$ - минимальная алгебра. По естественным свойствам гомологической алгебры (аналогичный аргумент используется при построении редуцированной бар-конструкции) будем называть решения уравнения Маурера-Картана нетривиальными, если они принадлежат идеалу $M(I)$, остальные решения будем называть тривиальными. В дальнейшем рассматриваются только нетривиальные решения.

ПРИМЕРЫ.

1) Решения гейзенберговского типа и идеал $H^{+}(\mathscr{A}) \cdot H^{+}(\mathscr{A})$.

Рассмотрим матрицу

$$
A=\left(\begin{array}{cccccc}
0 & \bar{a}_{1} & \bar{a}_{2} & \ldots & \bar{a}_{l} & c \\
0 & 0 & & & 0 & b_{1} \\
& & \ddots & & & b_{2} \\
& & & \ddots & & \vdots \\
0 & & \ldots & & 0 & b_{l} \\
0 & & \ldots & & 0 & 0
\end{array}\right)
$$

где $a_{i}, b_{j}, c \in I, a_{i j}=0, i, j>l+1$. Мы называем эту матрицу матрицей гейзенберговского типа, используя аналогию со структурой элементов алгебры Ли обобщенной групшы Гейзенберга. Попытаемся найти решение уравнения Маурера-Картана вида (6). Непосредственно из определения получаем, что $d a_{i}=$ $d b_{j}=0$ и если $\alpha_{i}, \beta_{j}$ - соответствуюшие классы когомологий, то обобщенное произведение Масси, определяемое $A$, имеет вид

$$
[\mu(A)]=-\sum_{i=1}^{n} \alpha_{i} \beta_{i} \in H^{+}(\mathscr{A}) \cdot H^{+}(\mathscr{A}) .
$$

Таким образом, мы видим, что каждый элемент идеала $H^{+}(\mathscr{A}) \cdot H^{+}(\mathscr{A})$ представляется произведением Масси гейзенберговского типа. В классической интерпретации (см. ниже) это - двуместное матричное произведение

$$
\left\langle\left(\bar{\alpha}_{1}, \ldots, \bar{\alpha}_{n}\right),\left(\begin{array}{c}
\beta_{1} \\
\vdots \\
\beta_{n}
\end{array}\right)\right\rangle .
$$

ОпРЕДЕЛЕНИЕ 4. Все обобщенные произведения Масси, принадлежащие идеалу $H^{+}(\mathscr{A}) \cdot H^{+}(\mathscr{A})$, называются вполне редуцируемыми. Обобщенное произведение Масси считается вполне нередущируемым, если соответствующий элемент имеет нетривиальньй образ при естественной проекции

$$
H^{*}(\mathscr{A}) \rightarrow H^{*}(\mathscr{A}) / H^{+}(\mathscr{A}) \cdot H^{+}(\mathscr{A})
$$


ЗАмЕчАниЕ. Как мы увидим ниже, между произведениями Масси возможны соотношения, позволяюшие сводить достаточно сложные произведения к более простым - в частности, к обычным произведениям. Так же, как и понятие нетривиальности матричных произведений Масси, введенное ниже, полная нередуцируемость характеризует "неупрошаемость" произведений Масси.

2) Тройнье (ординарные) произведения Масси.

Пусть замкнутые элементы $a \in \mathscr{A}^{p}, b \in \mathscr{A}^{q}$ и $c \in \mathscr{A}^{r}$ отвечают классам когомологий $\alpha, \beta$ и $\gamma$. Тройное произведение Масси $\langle\alpha, \beta, \gamma\rangle$ определено, если разрешимо уравнение Маурера-Картана для

$$
A=\left(\begin{array}{cccc}
0 & a & f & h \\
0 & 0 & b & g \\
0 & 0 & 0 & c \\
0 & 0 & 0 & 0
\end{array}\right)
$$

которое расписывается как

$$
d f=(-1)^{p} a \wedge b, \quad d g=(-1)^{q} b \wedge c
$$

Отсюда следует, что произведение $\langle\alpha, \beta, \gamma\rangle$ определено тогда и только тогда, когда

$$
\alpha \cup \beta=\beta \cup \gamma=0 \quad \text { в } H^{*}(\mathscr{A})
$$

Матрица $\mu(A)$ имеет вид (5) при $n=3$ и определяет произведение Масси $[\mu(A)]$ вида

$$
\langle\alpha, \beta, \gamma\rangle=[\tau]=\left[(-1)^{p+1} a \wedge g+(-1)^{p+q} f \wedge c\right] .
$$

Так как $f$ и $g$ определяются из (7) с точностью до замкнутых элементов из $\mathscr{A}^{p+q}$ и $\mathscr{A}^{q+r}$, то тройное произведение Масси $\langle\alpha, \beta, \gamma\rangle$ определяется с точностью до $\alpha \cdot H^{q+r}(\mathscr{A})+\gamma \cdot H^{p+q}(\mathscr{A})$.

3) п-местные ординарные произведения Масси [20].

Если $\alpha_{1}, \ldots, \alpha_{n} \in H^{*}(\mathscr{A})$, то их $n$-местное произведение Масси $\left\langle\alpha_{1}, \ldots, \alpha_{n}\right\rangle$ определено, если сушествуют такие их представители $a_{1}, \ldots, a_{n}$, для которых разрешимо уравнение Маурера-Картана с матрицей $A$ вида (4). При этом $\mu(A)$ примет вид (5) и $n$-местное (ординарное) произведение Масси определяется как $\left\langle\alpha_{1}, \ldots, \alpha_{n}\right\rangle=[\tau]$. Очевидно, при $n=3$ мы получаем уже разобранное в больших деталях (с указанием характера неоднозначности) тройное произведение Масси.

Начальные условия. Пусть $A$ - некоторое решение уравнения Маурера-Картана. Непосредственно из определения получаем, что на первой диагонали $A$ должны стоять только замкнутые элементы, т.е. $d a_{i+1}=0, i=1,2, \ldots$. На следующих диагоналях также могут появляться замкнутые элементы. Так, например, происходит в случае матричных произведений Масси (мы обсудим это ниже). Рассмотрим классы когомологий всех замкнутых элементов $A$ (или их часть) и назовем их начальными условиями соответствующей задачи Маурера-Картана.

Следующее утверждение (хорошо известное в классическом случае $n$-местных ординарных и $n$-местных матричных произведений Масси, см. [20], [21]) показывает, что множество гомологических классов $[\mu(A)]$ различных решений с данными начальными условиями зависит только от начальных условий. 
ПРЕДЛОЖЕНИЕ 1. Пусть $A$ - некоторое решение уравнения Маурера-Картана и $\mathscr{K}=\operatorname{Ker}$ А зафиксировано. Любое изменение әлемента $a_{i j}$ матрицы $A$ на $a_{i j}+d b$ допускает продолэсение $A^{\prime}$ такое, что

1) $A^{\prime}=A+(d b)_{i j} \bmod M^{j-i+1, *}$;

2) $A^{\prime}$ - решение уравнения Маурера-Картана $\bmod \mathscr{K}$;

3) $\left[\mu\left(A^{\prime}\right)\right]=[\mu(A)]$.

ДокАЗАТЕЛЬСтво. Прямое вычисление показьвает, что свойствам 1) и 2) удовлетворяет

$$
A^{\prime}=A+(d b)_{i j}+A \cdot(b)_{i j}-(\bar{b})_{i j} \cdot A .
$$

Эти же вычисления показывают, что

$$
\mu\left(A^{\prime}\right)=\mu(A)+d\left(\left(A \cdot(b)_{i j}-(\bar{b})_{i j} \cdot A\right) \cap \mathscr{K}\right),
$$

таким образом, 3) также выполнено. Предложение доказано.

Естественность обобшенных произведений Масси. Пусть $f: \mathscr{A} \rightarrow \mathscr{B}$ - отображение градуированных дифференциальных алгебр, оно индуцирует отображение $\widehat{f}: M(\mathscr{A}) \rightarrow M(\mathscr{B})$ по правилу

$$
\widehat{f}\left(\left(a_{i j}\right)_{i, j \geqslant 1}\right)=\left(f\left(a_{i j}\right)\right)_{i, j \geqslant 1} .
$$

Очевидно, что для любой матрицы $A \in M(\mathscr{A})$

$$
\widehat{f}(\operatorname{Ker} A) \subseteq \operatorname{Ker}(\widehat{f}(A))
$$

Таким образом, каждое решение $A$ уравнения Маурера-Картана на алгебре $\mathscr{A}$ переходит в решение $\widehat{f}(A)$ уравнения Маурера-Картана на алгебре $\mathscr{B}$. Тем самым мы получаем отображение обобшенных произведений Масси по правилу

$$
f^{*}([\mu(A)])=[\mu(\widehat{f}(A))] .
$$

Стандартные рассуждения гомологической алгебры показывают, что справедливо

ПРЕДЛОЖЕНИЕ 2. Если $f$-квазиизоморфизм дифференциальных алгебр, то отображение (8) взаимно однозначно на обобщенных произведениях Масси.

Для матричных произведений Масси соответствуюшее утверждение доказано в [21; теорема 1.5].

Отсюда вытекает, что если $\mathscr{M}=\mathscr{M}(\mathscr{A})$ - минимальная модель алгебры $\mathscr{A}$, то обобщенные произведения Масси в $H^{*}(\mathscr{A})$ можно вычислять непосредственно по минимальной модели.

2.4. Классические операции $-n$-местные ординарные и матричные произведения Масси. Здесь мы кратко изложим, как укладываются в описанную схему классические $n$-местные произведения Масси, введенные в 60-е годы в работах [20] и [21]. Надо отметить, что связь произведений Масси с уравнением Маурера-Картана впервые заметил Мэй [21], но эта аналогия не получила никакого развития.

Пусть $\alpha_{1}, \ldots, \alpha_{n}, \alpha_{i} \in H^{p_{i}}(\mathscr{A}), i=1, \ldots, n,-$ последовательность классов когомологий, и пусть $a_{i} \in \alpha_{i}-$ представляющие их коциклы из алгебры $\mathscr{A}$. 
ОПРЕДЕЛЕНИЕ 5. Подмножество $A=(a(i, j)), 1 \leqslant i \leqslant j \leqslant n,(i, j) \neq(1, n)$, элементов $\mathscr{A}$ назьвается определяющей системой для произведения $\left\langle a_{1}, \ldots, a_{n}\right\rangle$, если

1) $a(i, i)=a_{i}, i=1, \ldots, n$;

2) $a(i, j) \in \mathscr{A}^{p(i, j)+1}$, где $p(i, j)=\sum_{r=i}^{j}\left(p_{r}-1\right)$;

3) $d a(i, j)=\sum_{r=i}^{j-1} \bar{a}(i, r) \cdot a(r+1, j)$.

Если эти условия вьполнены, то

4) $(p(1, n)+2)$-мерный коцикл

$$
c(A)=\sum_{r=1}^{n-1} \bar{a}(1, r) a(r+1, n)
$$

назьвается коциклом определяющей системы $A$.

ОПРЕДЕЛЕНИЕ $6 . n$-мерное произведение $\left\langle a_{1}, \ldots, a_{n}\right\rangle$ считается определенным, если существует хоть одна определяющая система для него. Если это произведение определено, то $\left\langle\alpha_{1}, \ldots, \alpha_{n}\right\rangle$ состоит из всех классов когомологий $[c(A)] \in H^{p(1, k)+2}(\mathscr{A})$, где $A$ пробегает всевозможные определяюшие системы для $\left\langle a_{1}, \ldots, a_{n}\right\rangle$.

В таком виде эти определения появились в [20], укажем связь этих определений с приведенными в предыдушем параграфе.

Пусть $A$ - определяющая система для $\left\langle a_{1}, \ldots, a_{n}\right\rangle$, ее естественно расположить в матрицу, которую мы обозначим той же буквой

$$
A=\left(\begin{array}{cccccc}
0 & a(1,1) & a(1,2) & \ldots & a(1, n-1) & a(1, n) \\
0 & 0 & a(2,2) & \ldots & a(2, n-1) & a(2, n) \\
\ldots \ldots \ldots \ldots \ldots \ldots \ldots \ldots \ldots \ldots \ldots \ldots \ldots & \ldots \ldots \ldots \ldots \ldots \ldots \\
0 & 0 & 0 & \ldots & a(n-1, n-1) & a(n-1, n) \\
0 & 0 & 0 & \ldots & 0 & a(n, n) \\
0 & 0 & 0 & \ldots & 0 & 0
\end{array}\right)
$$

где в качестве $a(1, n)$ может фигурировать любой элемент $\mathscr{A}^{p(1, n)+2}$. Легко видеть, что условия 1)-3) определения 5 эквивалентны тому, что $A$ удовлетворяет уравнению Маурера-Картана. При этом матрица $-\mu(A)$ имеет ровно один ненулевой элемент, который и есть $c(A)$. Обратно, если $A$ - решение уравнения Маурера-Картана с начальньпи данными $a_{i+1}=a_{i}, i=1,2, \ldots, n$, то элементы этой матрицы задают определяющую систему для $\left\langle a_{1}, \ldots, a_{n}\right\rangle$.

Матричные произведения Масси были введены впервые, по-видимому, Мэем [21]. Поскольку матричные элементы в таких произведениях должны всегда оставаться однородньми элементами $\mathscr{A}$, то нам потребуется следующее определение умно жимих матриц.

ОПРЕДЕЛЕНИЕ 7. Пусть $N(I)$ - множество всевозможных конечных прямоугольных матриц, элементами которых являются однородные элементы из идеала аугментации $I$. Две матрицы $X, Y \in N(I)$ размеров $p \times q$ и $r \times s$ соответственно называются умножсимылми (в заданном порядке), если

1) $q=r$

2) $\operatorname{deg} x_{i j}+\operatorname{deg} y_{j k}$ не зависит от $j$ для всех $(i, k), 1 \leqslant i \leqslant p, 1 \leqslant k \leqslant s$. 
С каждой матрицей $X=\left(x_{i j}\right)_{\substack{i=1 \\ j=1}}^{p} \in N(I)$ связана целочисленная матрица $\mathscr{D}(X)=\left(\operatorname{deg} x_{i j}\right)$. Если $X$ и $Y$ - умножимые матрищы, то

$$
\mathscr{D}(X \cdot Y)=\mathscr{D}(X) * \mathscr{D}(Y)=\left(\operatorname{deg} x_{i j}+\operatorname{deg} y_{j k}\right)_{i k}
$$

Дальнейшее определение матричных произведений Масси полностью аналогично схеме определения ординарных произведений. Пусть $V_{1}, V_{2}, \ldots, V_{n}$ - некоторый набор матриц из $N\left(H^{+}(\mathscr{A})\right)$ таких, что $V_{i}$ и $V_{i+1}$ умножимы, $i=1, \ldots, n-1$. Рассмотрим матрицы $X_{i} \in N(I)$, состоящие из представляющих коциклов матриц $V_{i}$, $i=1, \ldots, n$, т.е.

$$
d X_{i}=0 \text { и }\left[X_{i}\right]=V_{i}, \quad i=1, \ldots, n .
$$

Последовательность $X_{i}, i=1, \ldots, n$, будет автоматически умножима и $\mathscr{D}\left(X_{i}\right)=$ $\mathscr{D}\left(V_{i}\right), i=1, \ldots, n$. Введем еще одно соглашение: если $K=\left(k_{i j}\right)$ - матрица с целочисленными элементами и $m$ - целое, то положим $K+m=\left(k_{i j}+m\right)$, т.е. к каждому матричному элементу добавляется $m$.

ОПРЕДЕЛЕНИЕ 8. Подмножество $A=(X(i, j)), 1 \leqslant i, j \leqslant n,(i, j) \neq(1, n)$, матриц из $N(I)$ назьвается определяющей системой матричного произведения $\left\langle X_{1}, \ldots, X_{n}\right\rangle$, если

1) $X(i, i)=X_{i}, i=1, \ldots, n$;

2) $\mathscr{D}(X(i, j))=\left(\mathscr{D}\left(X_{i}\right)-1\right) *\left(\mathscr{D}\left(X_{i+1}\right)-1\right) * \cdots *\left(\mathscr{D}\left(X_{j}\right)-1\right)+1$;

3) $d X(i, j)=\sum_{r=i}^{j-1} \bar{X}(i, r) \cdot X(r+1, j)$.

Если эти условия выполнены, то

4) матричньй коцикл

$$
c(A)=\sum_{r=1}^{n-1} \bar{X}(1, r) \cdot X(r+1, n)
$$

степени $\mathscr{D}(1, p)=\mathscr{D}\left(X_{1}\right) * \cdots * \mathscr{D}\left(X_{n}\right)-n+2$ называется коииклом определяющей системы $A$.

ОПРЕДЕЛЕНИЕ 9. $n$-местное матричное произведение $\left\langle X_{1}, \ldots, X_{n}\right\rangle$ считается определенным, если существует хотя бы одна определяюшая система для него. Если это произведение определено, то $\left\langle V_{1}, \ldots, V_{n}\right\rangle$ состоит из всех матричных классов когомологий $[c(A)]$, где $A$ пробегает всевозможные определяюшие системы для $\left\langle X_{1}, \ldots, X_{n}\right\rangle$.

В точности как и выше, каждой определяюшей матричной системе $A$ соответствует блочная матрица

$$
A=\left(\begin{array}{cccccc}
0 & X(1,1) & X(1,2) & \ldots & X(1, n-1) & X(1, n) \\
0 & 0 & X(2,2) & \ldots & X(2, n-1) & X(2, n) \\
\ldots \ldots & \ldots \ldots & \ldots \ldots \ldots \ldots \ldots \ldots \ldots \ldots \ldots \ldots \ldots \ldots \\
0 & 0 & 0 & \ldots & X(n-1, n-1) & X(n-1, n) \\
0 & 0 & 0 & \ldots & 0 & X(n, n) \\
0 & 0 & 0 & \ldots & 0 & 0
\end{array}\right)
$$


где $X(i, j)$ - матричные блоки из определения 8. При этом блок $X(1, n)$ принадлежит $\operatorname{Ker} A$ и может быть совершенно любым. Матрица $A$ удовлетворяет уравнению Маурера-Картана, и - $\mu(A)$ имеет ровно один ненулевой блок (на 1-й мультистроке и $n$-м мультистолбце), равный $c(A)-d X(1, n)$, т.е.

$$
-[\mu(A)]=[c(A)]
$$

Верно и обратное, если $A$ - решение уравнения Маурера-Картана с блочными начальными условиями, соответствующими матричньм классам когомологий $V_{1}$, $\ldots, V_{n}$, то $-[\mu(A)] \in\left\langle V_{1}, \ldots, V_{n}\right\rangle$.

Аналог предложения 1 для $n$-местных произведений был доказан в [20] для ординарного случая и в [21] для матричного.

ПРЕДЛОЖЕНИЕ 3. Пусть п-местное (матричное) произведение Масси $\left\langle V_{1}\right.$, $\left.\ldots, V_{n}\right\rangle$ определено, тогда его значения зависят только от (матричных) классов когомологий $V_{1}, \ldots, V_{n}$.

Степень неопределенности и нетривиальность произведений Масси. Матричные произведения Масси не являются формальным обобщением ординарных $n$-местных операций, а возникают вполне естественным образом, например, при попытке описать, какова неоднозначность данного $n$-местного произведения. Этот эффект практически не заметен для классических трехместных произведений $\left\langle v_{1}, v_{2}, v_{3}\right\rangle$, но уже в полной мере проявляется для четырехместных произведений. Дадим точные определения.

ОПРЕДЕЛЕНИЕ 10. Пусть $n$-местное матричное произведение Масси $\left\langle V_{1}\right.$, $\left.\ldots, V_{n}\right\rangle$ определено. Это произведение называется тривиальным, если $0 \in$ $\left\langle V_{1}, \ldots, V_{n}\right\rangle$.

В соответствии с определением 4 дадим следующее

ОПРЕДЕЛЕНИЕ 11. $n$-местное (матричное) произведение Масси $\left\langle V_{1}, V_{2}, \ldots, V_{n}\right\rangle$ называется вполне редуцируемым, если

$$
\left\langle V_{1}, \ldots, V_{n}\right\rangle \subset H^{+}(\mathscr{A}) \cdot H^{+}(\mathscr{A})
$$

в противном случае будем назьвать его нередуиируемым. Такое произведение будем называть сильно нередуцируемым, если

$$
\left\langle V_{1}, \ldots, V_{n}\right\rangle \cap H^{+}(\mathscr{A}) \cdot H^{+}(\mathscr{A})=\varnothing
$$

Далее предположим, что $\left\langle V_{1}, \ldots, V_{n}\right\rangle$ и $\left\langle W_{1}, \ldots, W_{n}\right\rangle$ определены и имеют одинаковые мультистепени, т.е. $\mathscr{D}\left(V_{1}, \ldots, V_{n}\right)=\mathscr{D}\left(W_{1}, \ldots, W_{n}\right)$. В этом случае положим:

$$
\begin{gathered}
\left\langle V_{1}, \ldots, V_{n}\right\rangle+\left\langle W_{1}, \ldots, W_{n}\right\rangle=\left\{x+y ; x \in\left\langle V_{1}, \ldots, V_{n}\right\rangle, y \in\left\langle W_{1}, \ldots, W_{n}\right\rangle\right\} \\
\lambda\left\langle V_{1}, \ldots, V_{n}\right\rangle=\left\{\lambda x ; x \in\left\langle V_{1}, \ldots, V_{n}\right\rangle\right\} .
\end{gathered}
$$


ОПРЕДЕЛЕНИЕ 12. Неопределенностью произведения Масси $\left\langle V_{1}, \ldots, V_{n}\right\rangle$ называется множество

$$
\operatorname{In}\left\langle V_{1}, \ldots, V_{n}\right\rangle=\left\{x-y ; x, y \in\left\langle V_{1}, \ldots, V_{n}\right\rangle\right\} .
$$

Таким образом, если $\left\langle V_{1}, \ldots, V_{n}\right\rangle$ тривиально, то

$$
\left\langle V_{1}, \ldots, V_{n}\right\rangle \subset \operatorname{In}\left\langle V_{1}, \ldots, V_{n}\right\rangle
$$

Следующее предложение, доказанное в [21], описывает неопределенность $n$-местных произведений через $(n-1)$-местные.

ПРЕДЛОЖЕНИЕ 4. Пусть произведение $\left\langle V_{1}, \ldots, V_{n}\right\rangle$ определено, тогда

$$
\begin{gathered}
\operatorname{In}\left\langle V_{1}, \ldots, V_{n}\right\rangle \subset \underset{\left(X_{1}, \ldots, X_{n-1}\right)}{\bigcup}\left\langle W_{1}, \ldots, W_{n-1}\right\rangle, \quad \text { əде } \\
W_{1}=\left(\begin{array}{ll}
V_{1} & X_{1}
\end{array}\right), \quad W_{k}=\left(\begin{array}{cc}
V_{k} & X_{k} \\
0 & V_{k+1}
\end{array}\right), \quad 2 \leqslant k \leqslant n-2, \\
W_{n-1}=\left(\begin{array}{c}
X_{n-1} \\
V_{n}
\end{array}\right) .
\end{gathered}
$$

Оббединение берется по всем наборам из $n-1$ матрич $X_{1}, \ldots, X_{n-1} \in$ $N\left(H^{*}(\mathscr{A})\right)$ таких, что $\mathscr{D}\left(X_{k}\right)=\mathscr{D}\left(V_{k}, V_{k+1}\right)-1$. Кроме того, для $n=3$ имеет место равенство

$$
\operatorname{In}\left\langle V_{1}, V_{2}, V_{3}\right\rangle=\bigcup_{\left(X_{1}, X_{2}\right)}\left\langle W_{1}, W_{2}\right\rangle=\bigcup_{\left(X_{1}, X_{2}\right)}\left(\bar{V}_{1} \cdot X_{2}+\bar{X}_{1} \cdot V_{3}\right)
$$

ЗАмЕчАниЕ. Свойства нетривиальности и нередуцируемости высших произведений Масси независимы. Произведение Масси может быть вполне редуцируемым, но нетривиальньм (см. п. 2.3). С другой стороны, если $n>3$, то $n$-местные произведения могут быть тривиальными, но не вполне редуцируемьми. Если $n=3$, то тривиальность всегда означает полную редуцируемость.

Строго определенные произведения Масси. Для получения всех элементов $n$ местного ординарного или матричного произведения Масси необходимо рассмотреть все определяющие системы для этого произведения, или, что эквивалентно, рассмотреть все решения уравнения Маурера-Картана с данными начальными условиями. Построение определяюших систем индуктивно, и этот процесс приводит нас, во-первых, к многозначности соответствующих произведений. Во-вторых, может возникнуть ситуация, когда уже частично построенную определяющую систему дальше продолжить нельзя. В отличие от многозначности, последний эффект не так широко известен, так как он не проявляется для 3-местных произведений, а лишь для $n$-местных, где $n \geqslant 4$. Потенциальная невозможность продолжения частично построенных определяющих систем приводит к следуюшему определению, впервые появившемуся в [22] (см. также [21]). 
ОПРЕДЕЛЕНИЕ 13. Пусть матричное произведение $\left\langle V_{1}, \ldots, V_{n}\right\rangle$ определено. Оно назьвается строго определенным, если каждое произведение

$$
\left\langle V_{i}, \ldots, V_{j}\right\rangle, \quad 1 \leqslant j-i \leqslant n-2
$$

содержит только нулевую матрищу.

Легко видеть, что тройные произведения строго определены. По многим причинам строго определенные произведения Масси более удобны для приложений. Доказательство следующего простого предложения можно найти в [21].

Лемма 2. Матричное произведение $\left\langle V_{1}, \ldots, V_{n}\right\rangle$ строго определено тогда и только тогда, когда кажсдая частично определенная определяющая система $\{X(i, j) ; j-i \leqslant k\}, 1 \leqslant k \leqslant n-2$, мохсет бить продолжена до определяющей системы $A$ произведения $\left\langle V_{1}, \ldots, V_{n}\right\rangle$.

2.5. Произведения Масси как препятствия к формальности. Наиболее ценное для этой работы свойство операций Масси выражает следующая

Теорема 4. Пусть дифференциальная градуированная алгебра А्A формальна. Тогда

а) обобщенные произведения Масси в $H^{*}(\mathscr{A})$ вполне приводимь;

б) п-местные матричные произведения Масси в $H^{*}(\mathscr{A})$ тривиальны при $n \geqslant 3$.

ДокаЗАТЕЛьство. Пусть $\mathscr{M}=(\mathscr{M}, d)$ - минимальная модель для $\mathscr{A}$ и $H^{*}=$ $H^{*}(\mathscr{A}, d)$. В силу формальности имеет место квазиизоморфизм

$$
h:(\mathscr{M}, d) \rightarrow\left(H^{*}, 0\right)
$$

Согласно предложению 2 мы можем вычислять обобшенные произведения Масси в $H^{*}(\mathscr{A})$ непосредственно в $(\mathscr{M}, d)$.

а) Пусть $A$ - произвольное решение уравнения Маурера-Картана и $[\mu(A)]-$ соответствуюшее произведение Масси. Применяя равенство (8), получим

$$
h^{*}([\mu(A)])=[\mu(\widehat{h}(A))]=-[\widehat{h}(\bar{A}) \cdot \widehat{h}(A)] \in M\left(H^{+} \cdot H^{+}\right) .
$$

Поскольку $h^{*}$ - изоморфизм, то $[\mu(A)] \in \mathscr{M}\left(H^{+} \cdot H^{+}\right)$, что доказывает а).

б) Пусть $\left\langle V_{1}, \ldots, V_{n}\right\rangle-n$-местное матричное произведение Масси. Из предложения 2 получаем равенство

$$
\left\langle V_{1}, \ldots, V_{n}\right\rangle=\left\langle V_{1}, \ldots, V_{n}\right\rangle_{H}
$$

где символ $\langle\cdot, \ldots, \cdot\rangle_{H}$ означает, что произведение Масси вычисляется в дифференциальной алгебре $\left(H^{*}, 0\right)$. Поскольку $d \equiv 0$, то легко видеть, что набор матриц $A=\left(X(i, i)=V_{i} ; X(i, j)=0 ; 1 \leqslant i, j \leqslant n, j-i>0:(i, j) \neq(1, n)\right)$ будет определяющей системой для $\left\langle V_{1}, \ldots, V_{n}\right\rangle_{H}$. Если $n \geqslant 3$, то $c(A)=0$ и, следовательно, $0 \in\left\langle V_{1}, \ldots, V_{n}\right\rangle_{H}$. Из равенства (10) вытекает утверждение б). Это завершает доказательство. Теорема доказана. 
СлЕДСтвИЕ 2. Если дифференциальная градуированная алгебра $(\mathscr{A}, d)$ имеет нередуцируемые или нетривиальные матричные произведения Масси, то она неформальна.

Приведенное простое соображение позволяет в ряде случаев "алгоритмически" строить препятствия к формальности.

ПримеР. Рассмотрим обобщенную группу Гейзенберга, т.е. группу матриц вида

$$
\left(\begin{array}{cccccc}
1 & x_{1} & x_{2} & \ldots & x_{n} & z \\
0 & 1 & 0 & \ldots & 0 & y_{1} \\
& & 1 & & & y_{2} \\
\vdots & & & \ddots & & \vdots \\
& & & & 1 & y_{n} \\
0 & & \ldots & & 0 & 1
\end{array}\right)
$$

где $x_{i}, y_{j}, z \in \mathbb{R}, 1 \leqslant i, j \leqslant n$. Возьмем в качестве равномерной решетки подгруппу целочисленных матриц и соответствуюшее нильмногообразие обозначим $X_{n}$. Минимальна модель $\mathscr{M}_{n}=\mathscr{M}\left(X_{n}\right)$ совпадает с комплексом, порожденным левоинвариантными 1-формами [17]. В координатах $(\bar{x}, \bar{y}, z)$ левоинвариантные 1-формы имеют базис

$$
\alpha_{i}^{+}=d x_{i}, \quad \alpha_{i}^{-}=d y_{i}, \quad 1 \leqslant i \leqslant n ; \quad \beta=-d z+\sum_{i=1}^{n} x_{i} d y_{i}
$$

Таким образом,

$$
\mathscr{M}_{n}=\Lambda\left(\alpha_{i}^{ \pm}, 1 \leqslant i \leqslant n, \beta ; d \alpha_{i}^{ \pm}=0, d \beta=\alpha_{1}^{+} \wedge \alpha_{1}^{-}+\cdots+\alpha_{n}^{+} \wedge \alpha_{n}^{-}\right) .
$$

Исходя из этого представления, можно вычислить $H^{*}\left(X_{n}, \mathbb{R}\right)$, но мы ограничимся лишь замечанием, что первые нетривиальные произведения Масси появляются в размерности $n+1$. Эти классы когомологий имеют вид

$$
\left[\beta \wedge \alpha_{1}^{\varepsilon_{1}} \wedge \alpha_{2}^{\varepsilon_{2}} \wedge \cdots \wedge \alpha_{n}^{\varepsilon_{n}}\right]
$$

где $\varepsilon_{i} \in\{ \pm 1\}, i=1, \ldots, n$. Элементы вида (11) не принадлежат идеалу $H^{+} \cdot H^{+}$, и легко видеть, что

$$
\begin{aligned}
& {\left[\beta \wedge \alpha_{1}^{\varepsilon_{1}} \wedge \cdots \wedge \alpha_{n}^{\varepsilon_{n}}\right]} \\
& \quad=\left\langle\left(\varepsilon_{1} \alpha_{1}^{-\varepsilon_{1}}, \varepsilon_{2} \alpha_{2}^{-\varepsilon_{2}}, \ldots, \varepsilon_{n} \alpha_{n}^{-\varepsilon_{n}}\right),\left(\begin{array}{c}
\alpha_{1}^{\varepsilon_{1}} \\
\alpha_{2}^{\varepsilon_{2}} \\
\vdots \\
\alpha_{n}^{\varepsilon_{n}}
\end{array}\right), \alpha_{1}^{\varepsilon_{1}} \wedge \alpha_{2}^{\varepsilon_{2}} \wedge \cdots \wedge \alpha_{n}^{\varepsilon_{n}}\right\rangle .
\end{aligned}
$$

Таким образом, классы когомологий (11) представляются нетривиальными и, следовательно, нередуцируемыми тройными матричньми произведениями Масси. 
2.6. Сферические коциклы и гомоморфизм надстройки. Пусть $X$ - конечный $C W$-комплекс. Элемент $q \in H^{k}(X, \mathbb{R})$ назовем сферическим, если существует отображение сферы

$$
f: S^{k} \rightarrow X
$$

такое, что $f^{*}(q) \neq 0$.

Поскольку $f^{*}\left(H^{+} \cdot H^{+}\right)=0$ для каждого отображения (12), то образ любого сферического коцикла не равен нулю при естественной проекции

$$
H^{+} \stackrel{r}{\rightarrow} H^{+} / H^{+} \cdot H^{+}
$$

Если $h_{*}: \pi_{*}(X) \rightarrow H_{*}(X, \mathbb{R})$ - вешественньй гомоморфизм Гуревича, то имеет место коммутативная диаграмма

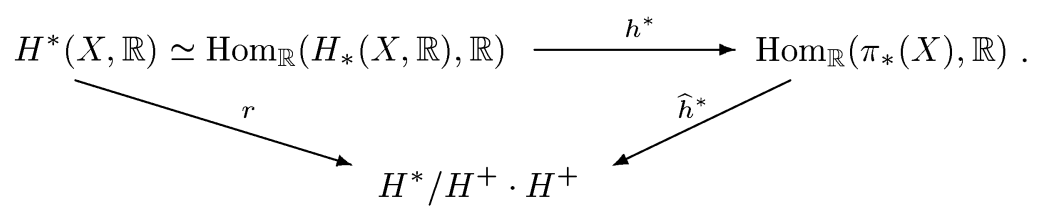

Выберем некоторое дополнительное к $\operatorname{Ker} h$ подпространство $S$ в $H^{*}(X, \mathbb{R})$ так, что $\left.r\right|_{S}$ - мономорфизм. Каждое такое подпространство $S$ назовем подпространством сферических образующих в $H^{*}(X, \mathbb{R})$.

Возникает естественньй вопрос: когда $H^{*}(X)$ порождено сферическими образующими? Из диаграммы (13) следует, что это имеет место тогда и только тогда, когда $\operatorname{rk} h_{*}=\operatorname{dim}\left(H^{*} / H^{+} \cdot H^{+}\right)$. В характеристике 0 этот вопрос достаточно подробно изучен. Различные условия, эквивалентные сферической порожденности, можно найти в [19]. Мы же приведем здесь только следующее

ПРЕДЛОЖЕНИЕ 5. Если пространство X формально, то $H^{*}(X)$ порождено сферическими образующими.

Доказательство этого предложения также можно найти в [19]. Заметим, что обращение этого утверждения неверно. Из приведенного утверждения мы получаем следуюшее препятствие к формальности.

СлЕДСтвИЕ 3. Если для $C W$-комплекса $X$

$$
\operatorname{rk} h_{*}<\operatorname{dim}\left(H^{*} / H^{+} \cdot H^{+}\right),
$$

где $h_{*}$ - гомоморфизм Гуревича, то $X$ неформально.

Подпространство сферических образуюших $S$ и подпространство, порожденное всевозможньми произведениями Масси (учитывая и обычные произведения), являются взаимно дополняющими подпространствами в $H^{*}(X, \mathbb{R})$. Чтобы придать этому утверждению четкий смысл, определим гомоморфизм когомологической надстройки

$$
\Omega^{*}: H^{*}(X, \mathbb{R}) \rightarrow H^{*-1}(\Omega X, \mathbb{R}),
$$

где $\Omega X$ - пространство петель над $X$. Обозначая, как обычно, через $[X, Y]$ множество гомотопических классов отображений $X$ в $Y$, можно определить (14) следуюшим образом:

$H^{n}(X, \mathbb{R}) \simeq[X, K(\mathbb{R}, n)] \stackrel{\Omega}{\rightarrow}[\Omega X, \Omega K(\mathbb{R}, n)]=[\Omega X, K(\mathbb{R}, n-1)] \simeq H^{n-1}(\Omega X, \mathbb{R})$. 
Альтернативное определение получается из рассмотрения расслоения $p: E X \stackrel{\Omega X}{\longrightarrow} X$ и точной когомологической последовательности пары $(E X, \Omega X)$. Имеют место гомоморфизмы

$$
H^{*-1}(\Omega X) \stackrel{\partial}{\longrightarrow} H^{*}(E X, \Omega X) \stackrel{p^{*}}{\longleftarrow} H^{*}(X, p t)
$$

причем $\partial-$ изоморфизм в силу стягиваемости $E X$. Таким образом, корректно определен гомоморфизм

$$
\partial^{-1} \circ p^{*}: H^{*}(X, p t) \rightarrow H^{*-1}(\Omega X)
$$

которьй совпадает с определенным выше, т.е. $\Omega^{*}=\partial^{-1} \circ p^{*}$.

Имеет место коммутативная диаграмма

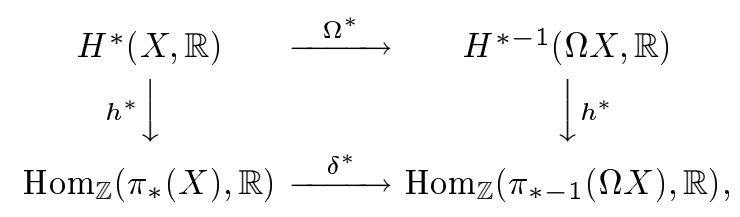

где $\delta^{*}$ индуцирован изоморфизмом $\partial^{-1}: \pi_{k-1}(\Omega X) \rightarrow \pi_{k}(X)$ точной гомотопической последовательности расслоения $E X \stackrel{\Omega X}{\longrightarrow} X$. Из этой диаграммы получаем

$$
\operatorname{Ker} h^{*}=\operatorname{Ker} \Omega^{*}
$$

Таким образом, имеет место разложение

$$
H^{+}(X, \mathbb{R})=S \oplus \operatorname{Ker} \Omega^{*}
$$

Среди специалистов считается доказанным следующее утверждение, принадлежащее Мэю (к сожалению, доказательство не было опубликовано).

Tеорема 5. Подпространство $\operatorname{Ker} \Omega^{*} \subset H^{+}(X, \mathbb{R})$ порожсдено всевозможными матричными произведениями Масси (учитывая и обычные произведения).

Отсюда вытекает полезное

СлЕДСТВИЕ 4. Для пространства $X$

$$
\operatorname{Ker} h_{*}<\operatorname{dim}\left(H^{+} / H^{+} \cdot H^{+}\right)
$$

тогда и только тогда, когда в $H^{*}(X, \mathbb{R})$ имеются нередуцируемые матричнве произведения Масси. 
2.7. Произведения Масси и спектральная последовательность Эйленберга-Мура. Здесь мы кратко изложим основные конструкции (в адаптированном для наших целей виде), относящиеся к спектральной последовательности Эйленберга-Мура. В заключение этого параграфа укажем связь когомологического гомоморфизма надстройки с последовательностью Эйленберга-Мура. Исчерпывающие алгебраические подробности можно найти в работе Смита [23] (см. также [19]).

Пусть, как и выше, $I$ - аугментированный идеал градуированной дифференциальной алгебры $(\mathscr{A}, d)$. Приведенная бар-конструкция для $\mathscr{A}$ определяется следующим образом. Положим

$$
B \mathscr{A}=\sum_{n \geqslant 0} \otimes^{n} I
$$

где тензорное произведение рассматривается над основным полем $k$. Если $a_{i} \in I$ - однородный элемент степени $p_{i}, i=1, \ldots, n$, то произведение $a_{1} \otimes a_{2} \otimes \cdots \otimes a_{n}$ обычно обозначается как $\left[a_{1}\left|a_{2}\right| \cdots \mid a_{n}\right]$. Модуль $B \mathscr{A}$ обладает естественной биградуировкой, которую мы получим, положив

$$
\operatorname{bideg}\left[a_{1}\left|a_{2}\right| \cdots \mid a_{n}\right]=\left(-n, \sum_{i=1}^{n} p_{i}\right)
$$

и общую степень определим формулой

$$
\operatorname{tdeg}\left[a_{1}\left|a_{2}\right| \cdots \mid a_{n}\right]=\sum_{i=1}^{n} p_{i}-n .
$$

Модуль $B \mathscr{A}$ автоматически получает структуру алгебры Хопфа с диагональю

$$
\Delta\left(\left[a_{1}\left|a_{2}\right| \cdots \mid a_{n}\right]\right)=\sum_{p=0}^{n}\left[a_{1}|\cdots| a_{p}\right] \otimes\left[a_{p+1}|\cdots| a_{n}\right]
$$

и умножением

$$
\left[a_{1}|\cdots| a_{k}\right] \cdot\left[a_{k+1}|\cdots| a_{k+n}\right]=\sum_{\sigma} \varepsilon_{\sigma}\left[a_{\sigma(1)}\left|a_{\sigma(2)}\right| \cdots \mid a_{\sigma(k+n)}\right]
$$

где сумма берется по всем упорядоченным перестановкам $(1, \ldots, k+n)$, т.е. $\sigma(1)<\sigma(2)<\cdots<\sigma(k)$ и $\sigma(k+1)<\sigma(k+2)<\cdots<\sigma(k+n)$. Знак $\varepsilon_{\sigma}$ перестановки $\sigma$ определяется обычньм способом через транспозиции, но каждая простая транспозиция $(i, i+1)$ соседних индексов имеет знак $(-1)^{\left(p_{i}-1\right)\left(p_{i+1}-1\right)}$.

На $B \mathscr{A}$ действуют два дифференциала - внутренний, определяемьй формулой

$$
d_{\mathscr{A}}\left(\left[a_{1}\left|a_{2}\right| \cdots \mid a_{n}\right]\right)=\sum_{i=1}^{n}(-1)^{i}\left[\bar{a}_{1}\left|\bar{a}_{2}\right| \cdots\left|\bar{a}_{i-1}\right| d a_{i}\left|a_{i+1}\right| \cdots \mid a_{n}\right],
$$

и комбинаторньй дифференциал

$$
\delta\left(\left[a_{1}\left|a_{2}\right| \cdots \mid a_{n}\right]\right)=\sum_{i=1}^{n}(-1)^{i}\left[\bar{a}_{1}\left|\bar{a}_{2}\right| \cdots\left|\bar{a}_{i-1} a_{i}\right| a_{i+1}|\cdots| a_{n}\right] .
$$


Эти дифференциалы биоднородны бистепеней $(1,0)$ и $(0,1)$ соответственно. Кроме того, $d_{\mathscr{A}} \delta+\delta d_{\mathscr{A}}=0$ и, следовательно, $\nabla=d_{\mathscr{A}}+\delta$ будет новьм дифференциалом бистепени $(1,1)$ на $B \mathscr{A}$. Алгебра Хопфа $B \mathscr{A}$, снабженная дифференциалом $\nabla$, называется бар-конструкиией для $(\mathscr{A}, d)$ и обозначается $(B A, \nabla)$.

Убываюшая фильтрация $\mathscr{F}_{n}(B \mathscr{A})=\left(\sum_{j \geqslant n} B \mathscr{A}^{j, *}\right)$ задается первой (внешней) градуировкой, соответствуюшая этой фильтрации спектральная последовательность назьвается спектральной последовательностью Эйленберга-Мура. Эта спектральная последовательность "живет" во втором квадранте. Хотя фильтрация в общем случае и бесконечна, член $\left\{E_{\infty}^{p, q}=\lim _{i} E_{i}^{p, q}\right\}^{p, q}$ корректно определен и присоединен к $H(B(\mathscr{A}), \nabla)$. Хорошо известно [24], что бар-конструкция, снабженная только комбинаторньм дифференциалом $\delta$, определяет гомологии $\operatorname{Tor}_{\mathscr{A}}^{* *}(k, k)$ с коэффициентами в $\mathscr{A}$-модуле $k$, где $\mathscr{A}$-действие определяется аугментацией $a(k)=\varepsilon(a) \cdot k$. Отсюда легко получаем, что член $E_{2}$ спектральной последовательности Эйленберга-Мура имеет вид

$$
E_{2}^{*, *} \simeq \operatorname{Tor}_{H^{*}(\mathscr{A})}^{*}(k, k) .
$$

Спектральная последовательность Эйленберга-Мура естественна по отношению к гомоморфизмам дифференциальных градуированных алгебр. Это означает, что если $\varphi:\left(\mathscr{A}, d_{\mathscr{A}}\right) \rightarrow\left(\mathscr{B}, d_{\mathscr{B}}\right)$ - гомоморфизм дифференциальных градуированных алгебр, то через отображение бар-конструкций мы получаем индуцированное отображение спектральных последовательностей

$$
\varphi^{*}:\left(E_{r}(\mathscr{A})^{*, *}, d_{r}\right) \rightarrow\left(E_{r}^{*, *}(\mathscr{B}), d_{r}\right), \quad r=1,2, \ldots
$$

Если алгебра $\mathscr{A}$ имеет топологический смысл, т.е. $(\mathscr{A}, d)=(\mathscr{E} *(X), d)$, где $X-$ гладкое односвязное многообразие, то для соответствующей последовательности Эйленберга-Мура будем иметь:

1) $E_{2}^{*, *} \simeq \operatorname{Tor}_{H^{*}(X, \mathbb{R})}^{*}(k, k)$;

2) $E_{r} \Rightarrow H^{*}(\Omega X, \mathbb{R})$.

Гомоморфизм когомологической надстройки следуюшим образом связан со спектральной последовательностью Эйленберга-Мура. Рассмотрим отображение $\Sigma: \mathscr{A} \rightarrow B(\mathscr{A})$, задаваемое формулой

$$
\Sigma(a)=[a] .
$$

Это отображение коммутирует с дифференциалами, поэтому мы получаем индуцированное отображение

$$
\Sigma^{*}: H^{+}(X) \rightarrow H(B(\mathscr{A}), \nabla) \simeq H^{*}(\Omega X, \mathbb{R}) .
$$

Следуюшее предложение, наверное, впервые доказано Муром [25], см. также [23].

ПРЕДЛОЖЕНИЕ 6. Имеет место равенство

$$
\Sigma^{*}=\Omega^{*} .
$$

Из формулы (15) легко видеть, что отображение $\Sigma^{*}$ пропускается через спектральную последовательность Эйленберга-Мура, так что мы имеем последовательность отображений

$$
\Sigma_{r}^{*}: H^{+}(X, \mathbb{R}) \rightarrow E_{r}^{-1, *} .
$$

В частности, $\Sigma_{1}^{*}: H^{+}(X, \mathbb{R}) \rightarrow E_{1}^{-1, *}=B^{-1}\left(H^{*}(X, \mathbb{R}), \delta\right)$ задается формулой $\Sigma_{1}^{*}(\alpha)=[\alpha]$ и существует естественная проекция

$$
\Sigma_{2}^{*}: H^{+}(X, \mathbb{R}) \rightarrow E_{2}^{-1, *}=\operatorname{Tor}_{H^{*}(X, \mathbb{R})}^{1}(k, k) \simeq H^{+}(X, \mathbb{R}) / H^{+} \cdot H^{+} .
$$


СЛЕДСТВИЕ 5. Если для пространства имеет место неравенство $\mathrm{rk} h_{*}<$ $\operatorname{dim} H^{+}(X, \mathbb{R}) / H^{+} \cdot H^{+}$, то соответствующая спектральная последовательность Эйленберга-Мура не стабилизируется в члене $E_{2}$.

\section{§3. Высшие операции Масси в симплектических многообразиях}

3.1. Симплектические многообразия и симплектические раздутия. Гладкое многообразие $X$ с заданной 2-формой $\omega$ на $X$ называется симплектическим, если форма $\omega$ удовлетворяет следуюшим двум условиям:

1) $\omega$ невырождена, т.е. для любого ненулевого вектора $\xi \in T_{x} X$ существует вектор $\eta \in T_{x} X$ такой, что $\omega(\xi, \eta) \neq 0$;

2) $d \omega=0$.

Такая форма $\omega$ назьвается симплектической.

Согласно теореме Дарбу в окрестности любой точки $x \in X$ существуют локальные координаты $x^{1}, \ldots, x^{2 n}$, в которых симплектическая форма имеет вид

$$
\omega=\sum_{j=1}^{n} d x^{j} \wedge d x^{j+n}
$$

Отсюда следует, что симплектическое многообразие $X$ должно иметь четную размерность и форма $\omega^{n}$ является формой объема:

$$
\omega^{n}=n ! d x^{1} \wedge \cdots \wedge d x^{n}
$$

Выберем на $X$ риманову метрику и зададим оператор $A$, действующий линейно в слоях касательного расслоения $T X$, формулой

$$
(A \xi, \eta)=g_{j k}(A \xi)^{j} \eta^{k}=\omega(\xi, \eta)
$$

Оператор $A$ кососимметричен, и следовательно, оператор $A^{*} A=-A^{2}$ симметричен и положителен. Возьмем положительный симметричньй квадратньй корень из $A^{*} A$ :

$$
Q=\sqrt{-A^{2}}>0
$$

и положим $J=A Q^{-1}$. Тогда

$$
J^{2}=-1
$$

и оператор $J$ задает почти комплексную структуру на $X$. Эта структура совместна с симплектической структурой: это означает, что форма

$$
\langle\xi, \eta\rangle=(\xi, J \eta)
$$

является эрмитовой и положительно определенной.

В дальнейшем мы ограничимся компактными симплектическими многообразиями, так как любое некомпактное почти комплексное многообразие имеет совместную симплектическую структуру [13]. В случае компактных многообразий условие $\left[\omega^{n}\right] \neq 0$ в $H^{2 n}(X, \mathbb{R})$ показывает, что не любое компактное комплексное многообразие имеет симплектическую структуру. 
Примерами симплектических многообразий являются кэлеровы многообразия, т.е. комплексные многообразия с эрмитовой метрикой $h_{j k} d z^{j} d \bar{z}^{k}$ такой, что форма

$$
\omega=h_{j k} d z^{j} \wedge d \bar{z}^{k}
$$

симплектична.

Другие примеры односвязных компактных симплектических многообразий размерности, большей четырех, получаются из известных с помощью симплектического расслоения [4], симплектического раздутия [13], [5] и расслоенной связной суммы [6]. Мы рассмотрим симплектические раздутия и прежде всего напомним их конструкцию.

Симплектическое раздутие. Пусть $f: Y \rightarrow X$ - симплектическое вложение, т.е. $\omega$ - симплектическая форма на $X$ и $f^{*} \omega$ - симплектическая форма на $Y$. Поэтому будем отождествлять $Y$ с подмногообразием $f(Y) \subset X$.

Каждой точке $x \in Y \subset X$ сопоставим подпространство $E_{x} \subset T_{x} X$, которое является симплектическим ортогональным дополнением к $T_{x} Y$ в $T_{x} X$ :

$$
\omega(\xi, \eta)=0 \quad \text { для } \xi \in T_{x} Y, \quad \eta \in E_{x} .
$$

Подпространства $E_{x}$ образуют векторное расслоение

$$
E \rightarrow Y
$$

изоморфное нормальному расслоению $Y$.

Так как $Y$ - симплектическое подмногообразие, то ограничения $\omega$ на $E_{x}$ невырождены и мы можем построить почти комплексную структуру $J_{x}$ на $E_{x}$, гладко зависящую от $x$ и совместную с $\left.\omega\right|_{E_{x}}$. Значит, структурная группа расслоения $E$ редуцируется к $U(k)=S O(2 k) \cap S p(k)$, где $k=\frac{1}{2}(\operatorname{dim} X-\operatorname{dim} Y)$.

Идентифицируем слои $E \mathrm{c} \mathbb{C}^{k}$ и рассмотрим другое расслоение

$$
\widetilde{E} \rightarrow Y
$$

слои которого изоморфны каноническому линейному расслоению $L \rightarrow \mathbb{C} P^{k-1}$ и которое имеет то же присоединенное главное $U(k)$-расслоение, что и расслоение $E \rightarrow Y$. Расслоение

$$
L \rightarrow \mathbb{C} P^{k-1},
$$

где

$$
L=\left\{(\xi, l) \in \mathbb{C}^{k} \times \mathbb{C} P^{k-1}: \xi \in l\right\},
$$

параметризует пары, состоящие из прямой $l \subset \mathbb{C}^{k}$ и точки $\xi \in l$. Проекция на базу имеет вид

$$
(\xi, l) \rightarrow l \in \mathbb{C} P^{k-1} .
$$

Структурная группа $U(k)$ действует на этом расслоении по правилу

$$
A \cdot(\xi, l)=(A \xi, A l) .
$$

Слои расслоения $E$, а значит, и расслоения $\widetilde{E}$ оснащены эрмитовой метрикой

$$
(\xi, \eta)=\omega(\xi, J \eta)
$$


и мы обозначим через $E_{r}$ и $\widetilde{E}_{r}$ подмногообразия $E$ и $\widetilde{E}$, выделенные условием: $|\xi| \leqslant r$.

Построим теперь симплектическое раздутие $\widetilde{X}$ многообразия $X$ вдоль $Y$. Для этого выберем замкнутую трубчатую окрестность $V$ подмногообразия $Y$ и отождествим ее с $E_{1}$. Удалим из $X$ внутренность $V$ и к полученному многообразию приклеим $\widetilde{E}_{1}$. Склейка задается изоморфизмом $\partial E_{1}=\partial \widetilde{E}_{1}=\partial V$. Полученное многообразие

$$
\widetilde{X}=\overline{(X \backslash V)} \bigcup_{\partial V} \widetilde{E}_{1}
$$

и называется симплектическим раздутием. Оно, фактически, является результатом послойного раздутия слоев $E_{x}$ в точках $\xi=0$.

Определена проекция

$$
\pi: \widetilde{X} \rightarrow X
$$

которая вне $Y$ является диффеоморфизмом

$$
\widetilde{X} \backslash \pi^{-1}(Y) \rightarrow X \backslash Y
$$

и над $Y$ является расслоением

$$
\pi^{-1}(Y) \rightarrow Y
$$

со слоем $\mathbb{C} P^{k-1}$.

Как показано в [13] и [5], на $\widetilde{X}$ сушествует симплектическая структура, совпадающая с $\pi^{*}(\omega)$ вне малой окрестности $\pi^{-1}(Y)$.

3.2. Выживание высших операций Масси. Основной целью этого пункта является доказательство следующего утверждения.

ТЕОрема А. Пусть односвязное симплектическое многообразие $X$ имеет нередуцируемое обобщенное произведение Масси размерности $k$. Тогда для любого симплектического подмногообразия $Y \subset X$ mакого, что $\operatorname{codim} Y>k$, соответствующее симплектическое раздутие $\widetilde{X}$ такосе имеет нередуцируемое обобщенное произведение Масси размерности $k$.

СЛЕДСТВИЕ 6. В условиях теоремы А оба многообразия - как $X$, так и $\widetilde{X}$ являются неформальнымми.

Из теоремы А и следствия 4 получаем

СЛЕДСТВИЕ 7. Пусть кольио когомологий односвязного симплектического многообразия $X$ обладает несферической образующей размерности $k$. Тогда симплектическое раздутие $\widetilde{X}$ вдоль любого симплектического подмногообразия $Y \subset X$ такого, что $\operatorname{codim} Y>k$, также обладает несферической $k$-мерной образующей в когомологиях и, следовательно, неформально.

Отсюда, используя следствие 3 , получаем

СлЕДСТВИЕ 8. Предположим, что односвязное симплектическое многообразие $Х$ удовлетворяет условию

$$
\operatorname{rk} h_{k}<\operatorname{dim} H^{k}(X) / H^{k}(X) \cap\left(H^{+}(X)\right)^{2} .
$$


Тогда симплектическое раздутие $\widetilde{X}$ вдоль любого симплектического подмногообразия $Y \subset X$ такого, что $\operatorname{codim} Y>k$, будет неформальным многообразием.

Перейдем к доказательству теоремы А. Пусть $\pi: \widetilde{X} \rightarrow X$ - естественная проекция. Согласно предположению существует элемент $u \in H^{k}(X)$, являюшийся обобшенньм нередуцируемьм произведением Масси. Покажем, что $\pi^{*}(u) \in H^{k}(\widetilde{X})$ также является обобщенным нередуцируемым произведением Масси. Пусть $A \in$ $M(\mathscr{E} *(X))$ - решение уравнения Маурера-Картана такое, что $u$ - матричный элемент матрицы $[d A-\bar{A} \cdot A]$. Согласно свойству естественности решений уравнения Маурера-Картана $\pi^{*}(A)$ является решением этого уравнения в $M(\mathscr{E} *(\widetilde{X}))$. Кроме того, соответствующий элемент матрицы

$$
\left[d \pi^{*}(A)-\overline{\pi^{*}(A)} \cdot \pi^{*}(A)\right]=\pi^{*}([d A-\bar{A} \cdot A])
$$

равен $\pi^{*}(u)$. Остается доказать нередуцируемость обобшенного произведения $\pi^{*}(u)$, что вытекает из следуюшего утверждения.

Лемма 3. Пусть $\pi: \widetilde{X} \rightarrow X-$ естественная проекиия симплектического раздутия $X$ вдоль $Y \subset X$. Индуцированное отображение

$$
\pi^{*}: \operatorname{Tor}_{1, k}^{H^{*}(X)}(\mathbb{R}, \mathbb{R}) \rightarrow \operatorname{Tor}_{1, k}^{H^{*}(\tilde{X})}(\mathbb{R}, \mathbb{R})
$$

является мономорфизмом при $k<\operatorname{codim} Y$.

Действительно, для алгебры $H=H^{*}(\mathscr{A})$ имеет место изоморфизм

$$
\operatorname{Tor}_{1, *}^{H}(k, k) \simeq H^{+} / H^{+} \cdot H^{+},
$$

следовательно, мономорфизм (17) в соответствуюших размерностях $k$ гарантирует, что $\pi^{*}(u) \neq 0 \bmod H^{+}(\widetilde{X}) \cdot H^{+}(\widetilde{X})$, т.е. нередуцируемость $\pi^{*}(u)$ как обобщенного произведения Масси.

ДокАЗАТЕЛЬСтво ЛЕммы 3. Пусть $V \subset X-$ трубчатая окрестность $Y$ и $\widetilde{V}-$ ее симплектическое раздутие вдоль $Y$. В этих обозначениях $\widetilde{X}=(X \backslash \dot{V}) \bigcup_{\partial \widetilde{V}} \widetilde{V}$, где $\dot{V}=V \backslash \partial V$ и края $\partial V$ и $\partial \widetilde{V}$ отождествлены естественным образом. Рассмотрим пространство

$$
\widehat{X}=X \bigcup_{\partial \widetilde{V}} \widetilde{V}=\widetilde{X} \bigcup_{\partial V} V
$$

Имеет место коммутативная диаграмма

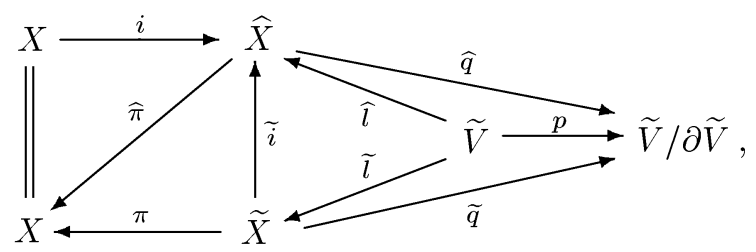

где $i, \widetilde{i}, \widehat{l}, \widetilde{l}$ - естественные вложения; $p, \widehat{q}, \widetilde{q}, \pi, \widehat{\pi}$ - естественные проекции, причем $\widehat{\pi}$-ретракция. 
Рассмотрим идеал $I=\operatorname{Ker} i^{*}$; так как $X$ является ретрактом $\widehat{X}$, то имеем мультипликативный изоморфизм

$$
H^{*}(\widehat{X}) \simeq H^{*}(X) \oplus I,
$$

где $H^{*}(X)$ идентифицирована со своим образом при ретракции $\widehat{\pi}^{*}$. Поскольку $I-$ идеал, из (19) вытекает, что

$$
\widehat{\pi}^{*}: H^{+}(X) /\left(H^{+}(X)\right)^{2} \rightarrow H^{+}(\widehat{X}) /\left(H^{+}(\widehat{X})\right)^{2}
$$

- мономорфизм.

Далее, из точной когомологической последовательности пары $(\widehat{X}, X)$ получаем, что $\widehat{q}^{*}$ отображает $H^{+}(\widetilde{V} / \partial \widetilde{V})$ изоморфино на $I$. Пусть $\widetilde{a} \in H^{2}(\widetilde{V} / \partial \widetilde{V})-$ проективный класс Тома. Используя расслоение

$$
r: \widetilde{V} \stackrel{\mathbb{C} P^{m}}{\longrightarrow} Y
$$

где $r$ - проекция, $m=\frac{1}{2} \operatorname{codim} Y$ и $\mathbb{C}^{m}=\mathbb{C} P^{m} \backslash D^{2 m}$, находим структуру $H^{+}(\widetilde{V} / \partial \widetilde{V})$ как $H^{*}(Y)$-модуля

$$
H^{+}(\widetilde{V} / \partial \widetilde{V}) \simeq \widetilde{a} H^{*}(Y) \oplus \widetilde{a}^{2} H^{*}(Y) \oplus \cdots \oplus \widetilde{a}^{m} H^{*}(Y) .
$$

Положим $\widehat{a}=\widehat{q}^{*}(\widetilde{a})$ и, применяя мономорфизм $\widehat{q}^{*}$, получим

$$
I \simeq \widehat{a} H^{*}(Y) \oplus \widehat{a}^{2} H^{*}(Y) \oplus \cdots \oplus \widehat{a}^{m} H^{*}(Y) .
$$

Расслоение $(21)$ определяет на $H^{*}(\widetilde{V})$ структуру $H^{*}(Y)$-модуля

$$
H^{*}(\widetilde{V}) \simeq H^{*}(Y) \oplus a H^{*}(Y) \oplus \cdots \oplus a^{m-1} H^{*}(Y),
$$

где $a \in H^{2}(\widetilde{V})$ - двумерньй класс, переходящий в образующую $\mathbb{C}^{m} \subset \widetilde{V}$. Заметим, что $a, \widehat{a}$ и $\widetilde{a}$ согласованы в смысле $a=\widehat{l}^{*}(\widehat{a})=p^{*}(\widetilde{a})$ и $\widetilde{a}=\widehat{q}^{*}(\widehat{a})$. Далее заметим, что разложения (22) и (23) согласованы, соответствующее отображение индуцировано вложением $\widehat{l}$. Наконец, положим

$$
I_{1}=\widehat{a} H^{*}(Y) \oplus \cdots \oplus \widehat{a}^{m-1} H^{*}(Y), \quad I_{2}=\widehat{a}^{m} H^{*}(Y)
$$

и покажем, что $\left.\widetilde{i}^{*}\right|_{I_{1}}-$ мономорфизм. Определим $H^{*}(Y)$-подмодуль $H^{*}(\widetilde{V})$, положив

$$
H_{+}^{*}(\widetilde{V})=a H^{*}(Y) \oplus a^{2} H^{*}(Y) \oplus \cdots \oplus a^{m-1} H^{*}(Y) .
$$

Как показано в [5], имеет место короткая точная последовательность модулей

$$
0 \longrightarrow H^{*}(X) \stackrel{\pi^{*}}{\longrightarrow} H^{*}(\widetilde{X}) \stackrel{\widetilde{l}^{*}}{\longrightarrow} H_{+}^{*}(\widetilde{V}) \longrightarrow 0
$$

Используя диаграмму (18), мы видим, что $\left.\widehat{l}^{*}\right|_{I_{1}}-$ изоморфизм и $\left.\widetilde{i}^{*}\right|_{I_{1}}-$ мономорфизм, расшепляющий $\widetilde{l}^{*}$.

Наконец, используя разложение $(19)$ и то, что $\widetilde{i}^{*}$ - мультипликативный изоморфизм в размерностях, не превосходящих $\operatorname{codim} Y$, получаем

$$
\left(H^{+}(\widetilde{X}) \cdot H^{+}(\widetilde{X})\right)^{k} \subseteq\left(H^{+}(X) \cdot H^{+}(X)\right) \oplus\left(i^{*}(I)\right)^{k}, \quad k<\operatorname{codim} Y .
$$

Отсюда заключаем, что

$$
\pi^{*}: H^{k}(X) / H^{k}(X) \cap\left(H^{+}(X)\right)^{2} \rightarrow H^{k}(\widetilde{X}) / H^{k}(\widetilde{X}) \cap\left(H^{+}(\widetilde{X})\right)^{2}
$$

- мономорфизм при $k<\operatorname{codim} Y$. Это доказывает лемму. 
ЗАмечАнИЕ. Для самого простого случая - 3-местных произведений Масси понятия нетривиальности и нередуцируемости очень близки. Небольшим видоизменением приведенного выше доказательства леммы 3 можно было бы получить следующее предложение.

ПРЕДЛОЖЕНИЕ 7. Пусть односвязное симплектическое многообразие $X$ имеет нетривиальное 3-местное произведение Масси в размерности $k$. Тогда для любого симплектического подмногообразия $Y \subset X$ такого, что $\operatorname{codim} Y>k$, соответствующее симплектическое раздутие $\widetilde{X}$ также имеет нетривиальное 3-местное произведение Масси размерности $k$.

3.3. Симплектические многообразия, обладающие произведениями Масси сколь угодно большого веса. Пусть $\left\langle X_{1}, \ldots, X_{k}\right\rangle$-некоторое $k$-местное матричное произведение Масси в $H^{*}(M)$. Будем говорить, что $\left\langle X_{1}, \ldots, X_{k}\right\rangle$ имеет вес cтрого $k$, если существует элемент $a \in\left\langle X_{1}, \ldots, X_{k}\right\rangle$, не принадлежащий линейному подпространству, порожденному всевозможными произведениями Масси $\left\langle Y_{1}, \ldots, Y_{l}\right\rangle$, где $l<k$. Как мы увидим ниже (замечание 3 ), в силу соотношений в когомологиях некоторые нетривиальные $k$-местные произведения Масси вполне могут выражаться через произведения Масси меньшего веса от совершенно иных элементов. Основньм результатом настояшего пункта является следуюшая

ТЕОРема 6. Для любого натурального $k$ существуют симплектические многообразия, обладающие матричными произведениями Масси веса строго $2 k$.

Для доказательства этого утверждения используем симплектические нильпотентные многообразия $M(2 n)$, введенные в нашей работе [2]. Напомним кратко их конструкцию, сохраняя обозначения этой работы.

Рассмотрим алгебру Витта $W(1)$ формальных векторных полей на прямой. Эта алгебра обладает базисом

$$
e_{i}=x^{i+1} \frac{d}{d x}, \quad i=-1,0,1, \ldots,
$$

в котором соотношения коммутации имеют вид

$$
\left[e_{i}, e_{j}\right]=(j-i) e_{i+j}, \quad i, j \geqslant-1 .
$$

Обозначая $\mathscr{L}(\cdot, \ldots, \cdot)$ линейную оболочку соответствующего набора векторов, рассмотрим подалгебры

$$
\mathscr{L}_{k}(1)=\mathscr{L}\left(e_{k}, e_{k+1}, \ldots\right), \quad k=1,2, \ldots
$$

Эти подалгебры задают естественную фильтрацию

$$
\cdots \subset \mathscr{L}_{1}(1) \subset \mathscr{L}_{0}(1) \subset \mathscr{L}_{-1}=W(1),
$$

полезную при изучении как самой $W(1)$, так и ее подалгебр и факторалгебр.

Далее рассмотрим серию конечномерных нильпотентных алгебр Ли

$$
\mathscr{V}_{n}=\mathscr{L}_{1}(1) / \mathscr{L}_{n+1}(1), \quad n=1,2, \ldots
$$


Соответствуюшие нильпотентные группы Ли обозначим $V_{n}, n=1,2, \ldots$ Из (25) получаем, что $\mathscr{V}_{n}$ - нильпотентная $n$-мерная алгебра Ли с базисом $\left\{e_{1}, \ldots, e_{n}\right\}$, в котором скобка Ли задается формулами

$$
\left[e_{i}, e_{j}\right]= \begin{cases}(j-i) e_{i+j}, & i+j \leqslant n \\ 0, & i+j>n\end{cases}
$$

Пользуясь нильпотентностью, мы можем отождествить $\mathscr{V}_{n}$ и $V_{n}$ как множества, а умножение $\times$ зададим по формуле Кемпбелла-Хаусдорфа. Другая интерпретация $V_{n}$ как группы полиномиальных преобразований прямой использована в недавней работе [26].

Формулы (26) показывают, что структурные константы $\mathscr{V}_{n}$ рациональны, следовательно, по теореме Мальцева [16] $V_{n}$ обладает равномерными решетками. Среди этих решеток выберем группы $\Gamma_{n}$, порожденные образуюшими $\left\{e_{1}, \ldots, e_{n}\right\}$ относительно умножения $\times$. Используя эти решетки, мы получаем последовательность нильмногообразий

$$
M(n)=V_{n} / \Gamma_{n}, \quad n=1,2, \ldots
$$

Заметим, что $V_{3}$ - трехмерная группа Гейзенберга $\mathscr{H}, M(3) \simeq \mathscr{H} / \mathscr{H}_{\mathbb{Z}}$, а $M(3) \times S^{1}$ - так называемое многообразие Кодаиры-Тёрстона.

Нильмногообразия $M(n), n \geqslant 3$, обладают рядом замечательных свойств, в частности, при четных $n$ они имеют естественную симплектическую структуру [2]. Пусть $\left\{\omega_{1}, \ldots, \omega_{n}\right\}$ - базис левоинвариантных 1-форм на $V_{n}$, двойственный базису $\left\{e_{1}, \ldots, e_{n}\right\}$. Из (26) получаем

$$
d \omega_{k}=(k-2) \omega_{1} \wedge \omega_{k-1}+(k-4) \omega_{2} \wedge \omega_{k-2}+\cdots .
$$

Далее, как показано в [2], левоинвариантная 2-форма

$$
\Omega_{2 m}=(2 m-1) \omega_{1} \wedge \omega_{2 m}+(2 m-3) \omega_{2} \wedge \omega_{2 m-1}+\cdots+\omega_{m} \wedge \omega_{m+1}
$$

задает левоинвариантную симплектическую структуру на $V_{2 m}$. При факторизации эта структура "опускается" на $M(2 m)$ и определяет целочисленную симплектическую структуру, которую мы обозначим тем же символом $\Omega_{2 m}$. Обозначим через $\left[\Omega_{2 m}\right] \in H^{2}(M(2 m), \mathbb{Z})$ класс когомологий этой формы. Теорема 6 вытекает из следуюшего утверждения.

ПРЕДЛОЖЕНИЕ 8. СИмплектический класс $\left[\Omega_{2 m}\right] \in H^{2}(M(2 m), \mathbb{Z})$ является 2 -местныц матричным произведением Масси веса строго $2 m$.

ДокАЗАТЕЛЬство. Пусть $\Lambda_{2 m}=\Lambda\left(\omega_{1}, \omega_{2}, \ldots, \omega_{2 m} ; d\right)$ - внешняя алгебра, где $d \omega_{1}=d \omega_{2}=0$ и $d \omega_{k}$ определены по формуле (27) для $k \geqslant 3$. Вложение

$$
\Lambda_{2 m} \rightarrow \mathscr{E}^{*}(M(2 m)),
$$

инициированное вложением левоинвариантных форм в комплекс всех форм на $M(2 m)$, согласно теореме Номидзу [17] является слабым изоморфизмом. Следовательно, мы можем найти произведения Массив $H^{*}(M(2 m))$, используя только $\Lambda_{2 m}$. Заметим, что $\Lambda_{2 m}$ изоморфна минимальной модели многообразия $M(2 m)$ [2]. 
Представим $\Omega_{2 m}$ в виде $2 m$-местного матричного произведения Масси. Имеет место включение

$$
\left[\Omega_{2 m}\right] \in\left\langle-m \omega_{1},-(m-1) \omega_{1}, \ldots,-2 \omega_{1},\left(-\omega_{1} 0\right),\left(\begin{array}{c}
\omega_{2} \\
\omega_{1}
\end{array}\right), 2 \omega_{1}, \ldots,(m-1) \omega_{1}, \omega_{1}\right\rangle .
$$

Чтобы доказать это включение, рассмотрим $(2 m+2) \times(2 m+2)$-матрицу $A$ вида

$$
\left(\begin{array}{cccccccc}
0 & -m \omega_{1} & (1-m) \omega_{2} & \ldots & (k-m) \omega_{1+k} & \ldots & (m-1) \omega_{2 m} & 0 \\
0 & 0 & (1-m) \omega_{1} & \ldots & (k-m) \omega_{k} & \ldots & (m-1) \omega_{2 m-1} & \omega_{2 m} \\
\ldots \ldots \ldots \ldots & \ldots \ldots \ldots \ldots \ldots \ldots & \ldots \ldots \ldots \ldots \ldots \ldots \ldots \ldots \ldots \ldots \ldots \ldots \ldots \\
0 & 0 & 0 & \ldots & 0 & \ldots & (m-1) \omega_{1} & \omega_{2} \\
0 & 0 & 0 & \ldots & 0 & \ldots & 0 & \omega_{1} \\
0 & 0 & 0 & \ldots & 0 & \ldots & 0 & 0
\end{array}\right)
$$

Прямым вычислением убеждаемся, что $A$ удовлетворяет уравнению МаурераКартана и, следовательно, задает некоторую определяющую систему произведения (28). Это же вычисление показьвает, что единственный ненулевой элемент матрицы $\bar{A} \wedge A-d A$, стоящий в первой строке и последнем столбце, имеет вид

$$
\begin{aligned}
-m \bar{\omega}_{1} \wedge \omega_{2 m}-(m-1) \bar{\omega}_{2} \wedge \omega_{2 m-1}-\ldots-\bar{\omega}_{m} \wedge \omega_{m+1}+\bar{\omega}_{m+2} \wedge \omega_{m-1} \\
+\cdots+(m-1) \bar{\omega}_{2 m} \wedge \omega_{1}=\sum_{i=1}^{m}[2(m-i)+1] \omega_{i} \wedge \omega_{2 m-i+1}=\Omega_{2 m}
\end{aligned}
$$

Последнее равенство доказьвает включение (28).

Наконец, покажем, что $\Omega_{2 m}$ не может быть выражена в виде какой-либо линейной комбинации $k$-местных произведений Масси с $k<2 m$. С этой целью заметим, что в действительности алгебра $\Lambda_{2 m}$ является биградуированной. Градуировки определяются на образующих

$$
\operatorname{deg}_{1}\left(\omega_{i}\right)=1, \quad \operatorname{deg}_{2}\left(\omega_{i}\right)=i, \quad i=1,2, \ldots, 2 m,
$$

и продолжаются по мультипликативности. Из формул (27) вытекает, что дифференциал $d$ имеет бистепень $(1,0)$. Заметим, что правила степени, использующиеся при определении многоместных матричных произведений Масси, введенные в п. 2.3, относятся только к $\operatorname{deg}_{1}$.

Кроме бистепени, алгебра $\Lambda_{2 m}$ имеет естественную возрастающую фильтрацию

$$
0=\mathscr{F}_{0} \subset \mathscr{F}_{1} \subset \cdots \subset \mathscr{F}_{2 m}=\Lambda_{2 m}
$$

где $\mathscr{F}_{k}=\Lambda\left(\omega_{1}, \ldots, \omega_{k}\right), k=1,2, \ldots, 2 m$. Эта фильтрация обладает следующими очевидными свойствами:

(i) $f(\alpha \wedge \beta) \leqslant \max (f(\alpha), f(\beta))$;

(ii) $f(d \alpha)<f(\alpha)$, причем если $\operatorname{deg}_{1}(\alpha)=1$, то $f(d \alpha)=f(\alpha)-1$.

Последнее свойство вытекает из (27).

Пусть

$$
\Omega_{2 m}=\sum_{s} \alpha_{s}\left\langle X_{1}^{(s)}, \ldots, X_{k(s)}^{(s)}\right\rangle, \quad k(s)<2 m, \quad \alpha_{s} \in \mathbb{R}
$$


где $X_{j}^{(s)}$ - замкнутыематрицы из элементов $\Lambda_{2 m}$. Поскольку $\operatorname{deg}_{1} \Omega=2$, то из правила степеней получим $\operatorname{deg}_{1} X_{j}^{(s)}=1$. Так как $X_{j}^{(s)}$ замкнута, то все ее элементы суть линейные комбинации $\omega_{1}$ и $\omega_{2}$, т.е.

$$
f\left(X_{j}^{(s)}\right) \leqslant 2 \text { для всех } s \text { и } j .
$$

Пусть $A^{(s)}=\left(X_{(i, j)}^{(s)}\right)$ - определяющая система, отвечающая $\left\langle X_{1}^{(s)}, \ldots, X_{k(s)}^{(s)}\right\rangle$. Снова применяя правило степеней для этого произведения, имеем $\operatorname{deg}_{1} X_{(i, j)}^{(s)}=1$ для всех $s, i, j$. Таким образом, из (30) и свойства (ii) вытекает $f\left(X_{(i, i+1)}^{(s)}\right) \leqslant 3$. Далее, применяя равенство 3 ) из определения 8 , по индукции получаем

$$
f\left(X_{(i, j)}^{(s)}\right) \leqslant j-i+2 .
$$

Последний шаг этого индуктивного процесса вместе с формулой 4) определения 8 дают

$$
f\left(c\left(A^{(s)}\right)\right) \leqslant k(s)
$$

где $c\left(A^{(s)}\right)$ - коцикл определяющей системы. С другой стороны, $f(\Omega(2 m))=2 m$, следовательно, равенство (29) невозможно, если $k(s)<2 m$ для всех $s$. Последнее противоречие доказывает предложение.

ЗАмЕчАния. 1) При доказательстве предложения 8 мы в явном виде предъявили матричное произведение Масси (28), которым представляется симплектический класс $\left[\Omega_{2 m}\right]$. Это произведение определено неоднозначно. Так, например, в $M(4)$ наряду с представлением (28) имеет место следующее включение:

$$
\left[\Omega_{4}\right] \in\left\langle\left(\begin{array}{ll}
0 & 6 \omega_{2}
\end{array}\right),\left(\begin{array}{cc}
\omega_{1} & \omega_{2} \\
0 & \omega_{1}
\end{array}\right),\left(\begin{array}{cc}
\omega_{1} & \omega_{2} \\
0 & \omega_{1}
\end{array}\right),\left(\begin{array}{l}
\omega_{2} \\
\omega_{1}
\end{array}\right)\right\rangle .
$$

Нахождение соответствуюшего решения уравнения Маурера-Картана мы оставляем читателю.

2) В многообразии $M(4)$ симплектический класс разлагается в сумму двух классов когомологий

$$
\left[\Omega_{4}\right]=\left[3 \omega_{1} \wedge \omega_{4}\right]+\left[\omega_{2} \wedge \omega_{3}\right]
$$

Несложно проверить, что первое слагаемое представляется 4-местным произведением Масси, например

$$
\left[3 \omega_{1} \wedge \omega_{4}\right] \in\left\langle 6 \omega_{2}, \omega_{1}, \omega_{1}, \omega_{1}\right\rangle
$$

и имеет вес строго 4. Второе слагаемое может быть представлено в виде

$$
\left[\omega_{2} \wedge \omega_{3}\right] \in\left\langle-\omega_{1}, \omega_{2}, \omega_{2}\right\rangle
$$

и имеет вес строго 3.

3) При $m>2$ оба класса $\left[3 \omega_{1} \wedge \omega_{4}\right]$ и $\left[\omega_{2} \wedge \omega_{3}\right]$ остаются ненулевьми в $H^{2}(M(2 m))$, но в силу равенства

$$
d \omega_{5}=\Omega_{4}=3 \omega_{1} \wedge \omega_{4}+\omega_{2} \wedge \omega_{3}
$$

их сумма равна нулю. Таким образом, в $H^{*}(M(2 m)), m>2$, мы получаем нетривиальное соотношение между 4-местным произведением Масси (31) и 3-местным (32). Следовательно, элемент $\left[3 \omega_{1} \wedge \omega_{4}\right] \in H^{2}(M(2 m))$ перестает быть классом когомологий веса строго 4 при $m>2$. 
3.4. Наследование высших операций Масси. Пусть, как и вьше, $(X, \omega)$ симплектическое многообразие и $Y \subset X$ - некоторое симплектическое подмногообразие. В этом пункте мы получим достаточные условия, при которых нетривиальные высшие матричные произведения Масси в $H^{*}(Y)$ наследуются при раздутии, т.е. порождают нетривиальные матричные произведения в когомологиях симплектического раздутия $H^{*}(\widetilde{X})$.

Рассмотрим матричные классы когомологий $S_{1}, \ldots, S_{n} \in N\left(H^{+}(\widetilde{X})\right)$, и пусть произведение $\left\langle S_{1}, \ldots, S_{n}\right\rangle$ определено и нетривиально. Наша цель - найти подходящее $n$-местное произведение Масси $\left\langle\widetilde{S}_{1}, \ldots, \widetilde{S}_{n}\right\rangle, \widetilde{S}_{i} \in N\left(H^{+}(Y)\right)$, нетривиальное в симплектическом раздутии $X$ вдоль $Y \subset X$. Если $n \geqslant 5$ и матричные классы $S_{i}, 1 \leqslant i \leqslant n$, a priori произвольны, то добиться этого эффективным способом достаточно проблематично даже при симплектическом вложении $Y \subset \mathbb{C} P^{N}$, где $N$ сколь угодно велико. Это объясняется структурой произведений Масси при $n \geqslant 5$ и возможным наличием "паразитических" соотношений в $H^{*}(Y)$ между произведениями Масси различных порядков. Тем не менее имеются два важных случая, которые мы рассмотрим ниже:

1) матрицы $S_{i}$ одномерны, т.е. $S_{i} \in N\left(H^{1}(Y)\right), 1 \leqslant i \leqslant n, n$ произвольно;

2 ) матрицы $S_{i}$ произвольны, $1 \leqslant i \leqslant n, n=3,4$.

Сформулируем основные результаты этого пункта.

Теорема В. Пусть симплектическое многообразие $(Y, \omega)$ имеет нетривиальное матричное п-местное произведение Масси $\left\langle S_{1}, \ldots, S_{n}\right\rangle$, где $S_{i} \in$ $N\left(H^{1}(Y)\right), 1 \leqslant i \leqslant n$. Тогда для любого симплектического вложсения $Y \subset X$ коразмерности не меньше $2(n+1)$ соответствующее симплектическое раздутие $\widetilde{X}$ имеет нетривиальное п-местное произведение Масси $\left\langle\widetilde{S}_{1}, \ldots, \widetilde{S}_{n}\right\rangle$, əде $\widetilde{S}_{i} \in N\left(H^{3}(\widetilde{X})\right), 1 \leqslant i \leqslant n$.

СлеДСТВИЕ 9. Для любого натурального $k$ существует односвязное симплектическое многообразие $X$ размерности $6 k+2$, имеющее нетривиальное $2 k$-местное матричное произведение Масси, лежащее в $H^{4 k+2}(X)$.

Действительно, рассмотрим симплектическое многообразие $M(2 k)$, определенное в п. 3.3. Пусть $f: M(2 k) \rightarrow \mathbb{C} P^{3 k+1}$ - симплектическое вложение, существуюшее по теореме Громова-Тышлера [27]. Теперь следствие непосредственно вытекает из предложения 8 и теоремы $\mathrm{B}$, примененной к паре $M(2 k) \subset \mathbb{C} P^{3 k+1}$. Следствие 9 установлено.

Теорема С. Пусть симплектическое многообразие $(Y, \omega)$ имеет нетривиальное тройное матричное произведение Масси. Тогда для любого симплектического вложения $Y \subset X$ коразмерности не меньше 8 соответствующее симплектическое раздутие $\widetilde{X}$ имеет нетривиальное тройное матричное произведение Масси.

Далее нам будет полезно следуюшее

ОПРеДЕЛЕниЕ 14. Пусть $\mathscr{A}$ - дифференциальная градуированная алгебра, $H \in$ $N(\mathscr{A})$ - матрица. Верхней степенью $H$ называется число

$$
\operatorname{sdeg} H=\max _{i, j} \operatorname{deg} h_{i j},
$$

где $h_{i j} \in \mathscr{A}$ суть матричные элементы $H$. 
ТЕОРема D. Пусть симплектическое многообразие $(Y, \omega)$ имеет сильно нередуцируемое четверное матричное произведение Масси $\left\langle S_{1}, S_{2}, S_{3}, S_{4}\right\rangle$. Тогда для любого симплектического вложения $Y \subset X$ такого, что

$$
\operatorname{codim} Y>2 \operatorname{sdeg}\left\langle S_{1}, S_{2}, S_{3}, S_{4}\right\rangle
$$

соответствующее симплектическое раздутие $\widetilde{X}$ обладает нетривиальным четверным матричным произведением Масси.

Перейдем к доказательствам этих теорем. Первая часть доказательства состоит в построении по имеюшемуся матричному произведению $\left\langle S_{1}, \ldots, S_{n}\right\rangle$ в $H^{*}(Y)$ соответствуюшего произведения $\left\langle\widetilde{S}_{1}, \ldots, \widetilde{S}_{n}\right\rangle$ в $H^{*}(\widetilde{X})$. Эта часть доказательства единообразна для всех теорем.

Пусть $S_{1}, \ldots, S_{n} \in N\left(H^{*}(Y)\right)$ - умножимые матрицы такие, что произведение Масси $\left\langle S_{1}, \ldots, S_{n}\right\rangle$ определено. Если $\pi: \widetilde{V} \rightarrow V \sim Y$ - естественная проекция, то $\pi^{*}: H^{*}(Y) \rightarrow H^{*}(\widetilde{V})$ - мономорфизм и мы не будем в дальнейшем делать различия между элементами $S_{i}$ и $\pi^{*}\left(S_{i}\right), 1 \leqslant i \leqslant n$. Рассмотрим матричные классы $a \cup S_{i} \in$ $N\left(H^{*}(\widetilde{V})\right), 1 \leqslant i \leqslant n$, где $a \in H^{2}(\widetilde{V})$ имеет тот же смысл, что и в п. 3.2. Как было показано в п. 3.2 ,

$$
p^{*}: H^{*}(\widetilde{V} / \partial \widetilde{V}) \rightarrow H^{*}(\widetilde{V})
$$

отображает изоморфино $\widetilde{a} \otimes H^{*}(Y) \subset H^{*}(\widetilde{V} / \partial \widetilde{V})$ на соответствующий подмодуль $a \cup H^{*}(Y) \subset H^{*}(\widetilde{V})$, где $p^{*}(\widetilde{a})=a$. Таким образом, корректно определены элементы $S_{i}^{\prime} \in N\left(H^{*}(\widetilde{V})\right)$ такие, что $p^{*}\left(S_{i}^{\prime}\right)=a \cup S_{i}, 1 \leqslant i \leqslant n$. Наконец, положим

$$
\widetilde{S}_{i}=\widetilde{q}^{*}\left(S_{i}^{\prime}\right), \quad 1 \leqslant i \leqslant n,
$$

и заметим, что в силу коммутативности диаграммы (18)

$$
\widetilde{l}^{*}\left(\widetilde{S}_{i}\right)=a \cup S_{i}=p^{*}\left(S_{i}^{\prime}\right), \quad 1 \leqslant i \leqslant n
$$

Доказательство теорем вытекает теперь из следующего утверждения.

Лемма 4. В условиях теорем В, С и D матричное произведение Масси $\left\langle\widetilde{S}_{1}, \ldots, \widetilde{S}_{n}\right\rangle$ определено и нетривиально.

В силу естественности произведений Масси и коммутативности диаграммы (18) эта лемма вытекает из двух утверждений.

Лемма 5. В условиях теорем В, С и D матричное произведение Масси $\left\langle S_{1}^{\prime}, \ldots, S_{n}^{\prime}\right\rangle$ определено.

Лемма 6. В условиях теорем В, С и D матричное произведение Масси $\left\langle a \cup S_{1}, \ldots, a \cup S_{n}\right\rangle$ нетривиально.

Заметим, что определенность последнего произведения Масси очевидна. 
ДокаЗАТЕЛЬСТво ЛЕммы 5. Пусть $A=(X(i, j)), 1 \leqslant i \leqslant j \leqslant n,(i, j) \neq$ $(1, n)$, - определяюшая система для произведения $\left\langle S_{1}, \ldots, S_{n}\right\rangle$. Имеем $X(i, j) \in$ $N\left(\mathscr{E}^{+}(Y)\right)$ и $[X(i, i)]=S_{i}, 1 \leqslant i \leqslant n$. Обозначим через $X^{\prime}(i, j)$ матричные дифференциальные формы $\pi^{*}(X(i, j)), 1 \leqslant i \leqslant j \leqslant n$. Далее, пусть $\alpha-2$-форма, представляющая класс $a \in H^{2}(\widetilde{V})$. Форму $\alpha$ можно выбрать так, что $\left.\alpha\right|_{U}=0$, где $U$ - малая трубчатая окрестность $\partial \widetilde{V}$. Действительно, если $j: \partial \widetilde{V} \rightarrow \widetilde{V}$ - вложение, то класс $a$ выбран так, что $j^{*}(a)=0$, т.е. $\alpha=d \beta$ на $U^{\prime}$, где $U^{\prime} \supset U$-некоторая трубчатая окрестность, содержащая $U$. Взяв обрезающую функцию $\varphi$, равную 0 на $U$ и 1 вне $U^{\prime}$, рассмотрим форму $\alpha_{1}=d(\varphi \beta)$ на $U^{\prime}$ и $\alpha_{1}=\alpha$ вне $U^{\prime}$. Поскольку $\alpha_{1} \sim \alpha$, то мы получаем представляюшую $a$ форму с нужными свойствами.

Легко видеть, что набор матричных форм

$$
\left(X^{\prime \prime}(i, j)=\alpha^{j-i+1} \wedge X^{\prime}(i, j)\right), \quad 1 \leqslant i \leqslant j \leqslant n,
$$

является определяющей системой для произведения $\left\langle a \cup S_{1}, \ldots, a \cup S_{n}\right\rangle$. Поскольку $\left.X^{\prime \prime}(i, j)\right|_{U} \equiv 0,1 \leqslant i \leqslant j \leqslant n$, то эту систему форм можно продолжить нулем на $\widetilde{V} \bigcup_{\partial \widetilde{V}} C \partial \widetilde{V} \simeq \widetilde{V} / \partial \widetilde{V}$. Таким образом мы получим систему симплициальных форм на $\widetilde{V} / \partial \widetilde{V}$, сохраним для них прежнее обозначение $A^{\prime \prime}=\left(X^{\prime \prime}(i, j)\right), 1 \leqslant i \leqslant j \leqslant n$. Из построения видно, что $A^{\prime \prime}$ будет определяющей системой для произведения $\left\langle S_{1}^{\prime}, \ldots, S_{n}^{\prime}\right\rangle$. Это завершает доказательство леммы 5 .

Доказательство леммы 6 распадается на три случая, из которых следуют теоремы $\mathrm{B}, \mathrm{C}$ и $\mathrm{D}$.

Лемма 6В. В условиях теоремь В матричное произведение Масси $\left\langle a \cup S_{1}\right.$, $\left.\ldots, a \cup S_{n}\right\rangle$ нетривиально.

ДокАЗАТЕльство. В отличие от предыдущего доказательства здесь удобнее проводить рассуждения не в алгебре форм, а в минимальных алгебрах. Пусть $\left(\mathscr{M}_{Y}, d_{Y}\right)$ - минимальная модель $Y$ и $m=\frac{1}{2} \operatorname{codim} Y$. Тогда минимальная модель для $\widetilde{V}$ устроена следующим образом (см. [2]):

$$
(\mathscr{M}, d) \simeq \mathscr{M}_{Y} \bigotimes_{d}\{x, y\}
$$

где $\operatorname{deg} x=2, \operatorname{deg} y=2 m-1,\left.d\right|_{\mathscr{M}_{Y}}=d_{Y}, d x=0$,

$$
d y=x^{m}+c_{1} \wedge x^{m-1}+c_{2} \wedge x^{m-2}+\cdots+c_{m-1} \wedge x+c_{m},
$$

$c_{i} \in \mathscr{M}_{Y}, 1 \leqslant i \leqslant m$. Заметим, что $[x]=a$.

Выберем в каждом классе когомологий $S_{i}, 1 \leqslant i \leqslant n$, какой-либо представитель $R_{i} \in N\left(\mathscr{M}_{Y}^{+}\right),\left[R_{i}\right]=S_{i}, 1 \leqslant i \leqslant n$. Таким образом,

$$
\left[x \wedge R_{i}\right]=a \cup S_{i}, \quad 1 \leqslant i \leqslant n .
$$

Предположим, что рассматриваемое произведение Масси тривиально, т.е.

$$
0 \in\left\langle a \cup S_{1}, \ldots, a \cup S_{n}\right\rangle
$$


и $A=(X(i, j)), 1 \leqslant i \leqslant j \leqslant n,-$ соответствуюшая определяющая система. Поскольку все матрицы $S_{i}, 1 \leqslant i \leqslant n$, состоят только из одномерных классов когомологий, то согласно правилу степеней имеем

$$
\operatorname{deg} X(i, j)=2(j-i)+3, \quad 1 \leqslant i \leqslant j \leqslant n, \quad(i, j) \neq(1, n)
$$

Здесь все матрицы составлены из элементов одной степени, поэтому степень матрицы корректно определена.

Разложим матрицы $X(i, j)$ по степеням $x$

$$
X(i, j)=\sum_{l=0}^{j-i} x^{l} \wedge X_{l}(i, j)+x^{j-i+1} \wedge X_{j-i+1}(i, j)
$$

где $X_{l}(i, j) \in \mathscr{M}_{Y}$. Поскольку $X(i, i)=x \wedge R_{i}, 1 \leqslant i \leqslant n$, то

$$
X_{1}(i, i)=R_{i} \quad\left(X_{0}(i, i)=0\right), \quad 1 \leqslant i \leqslant n .
$$

По условию теоремы В мы имеем $j-i+1 \leqslant n-1<m$ для всех $X(i, j)$. Следовательно, из соотношения

$$
d X(i, j)=\sum_{r=i}^{j-1} \overline{X(i, r)} \wedge X(r+1, j)
$$

и (34) получим

$$
d X_{j-i+1}(i, j)=\sum_{r=i}^{j-1} \overline{X_{r-i+1}(i, r)} \wedge X_{r+1}(r+1, j)
$$

Последнее равенство вместе с (36) означает, что $A_{1}=\left(X_{j-i+1}(i, j)\right), 1 \leqslant i \leqslant$ $j \leqslant n,-$ определяющая система для произведения $\left\langle S_{1}, \ldots, S_{n}\right\rangle$. Аналогичное вычисление с учетом $(35)$, примененное к циклу определяющей системы $c(A)$, дает

$$
\begin{aligned}
c(A) & =\sum_{r=1}^{n-1} \overline{X(1, r)} \wedge X(r+1, n) \\
& =x^{n} \wedge \sum_{r=1}^{n-1} \overline{X_{r}(1, r)} \wedge X_{n-r}(r+1, n)+\cdots=x^{n} \wedge c\left(A_{1}\right)+\cdots,
\end{aligned}
$$

где многоточие означает члены, имеющие по $x$ степень меньше $n$.

Так как $n<m$, то последнее равенство означает, что $[c(A)]=0$ влечет $\left[c\left(A_{1}\right)\right]=0$, т.е. произведение $\left\langle S_{1}, \ldots, S_{n}\right\rangle$ тривиально. Мы приходим к противоречию и, следовательно, лемма 6 В и теорема В доказаны.

Лемма 6С. В условиях теоремь С матричное произведение Масси $\left\langle a \cup S_{1}\right.$, $\left.a \cup S_{2}, a \cup S_{3}\right\rangle$ нетривиально. 
ДокаЗАТЕльство. Пусть $A_{1}=(R(i, j)), 1 \leqslant i \leqslant j \leqslant 3, R(i, j) \in \mathscr{M}_{Y},-$ некоторая определяюшая система для произведения $\left\langle S_{1}, S_{2}, S_{3}\right\rangle$. Тогда $A=\left(x^{j-i+1} \wedge\right.$ $R(i, j)), 1 \leqslant i \leqslant j \leqslant 3$, будет определяющей системой произведения $\left\langle a \cup S_{1}, a \cup S_{2}\right.$, $\left.a \cup S_{3}\right\rangle$. Очевидно, что $c(A)=x^{3} \wedge c\left(A_{1}\right)$, т.е. $[c(A)]=a^{3} \cup\left[c\left(A_{1}\right)\right]$.

Неопределенность тройного произведения Масси устроена очень просто (см. предложение 4), и мы получаем, что любой элемент $u \in\left\langle a \cup S_{1}, a \cup S_{2}, a \cup S_{3}\right\rangle$ имеет вид

$$
u=[c(A)]+H \cup a \cup S_{3}+a \cup \bar{S}_{1} \cup K,
$$

где $H, K \in N\left(H^{+}(\widetilde{V})\right)$ - произвольные матрицы соответствующих размеров и мультистепеней.

Поскольку $H^{*}(\widetilde{V})$ является свободным $H^{*}(Y)$-модулем с базисом $1, a, \ldots, a^{m-1}$, то разлагая $a \cup H$ и $a \cup K$ по этому базису, из (37) получим

$$
u=a^{3} \cup\left[c\left(A_{1}\right)\right]+\left(\sum_{i=0}^{m-1} a^{i} \cup H_{i}\right) \cup S_{3}+\bar{S}_{1} \cup\left(\sum_{i=0}^{m-1} a^{i} \cup K_{i}\right) .
$$

Далее, имеем $S_{i} \in N\left(H^{+}(Y)\right)$ и $m \geqslant 4$, следовательно, при $u=0$ из (38) необходимо вытекает

$$
\left[c\left(A_{1}\right)\right]+H_{3} \cup S_{3}+\bar{S}_{1} \cup K_{3}=0 .
$$

Последнее равенство означает, что $0 \in\left\langle S_{1}, S_{2}, S_{3}\right\rangle$ вопреки предположению. Лемма и вместе с ней теорема $\mathrm{C}$ доказаны.

ЛЕмма 6D. В условиях теоремы D матричное произведение Масси $\left\langle a \cup S_{1}\right.$, $\left.a \cup S_{2}, a \cup S_{3}, a \cup S_{4}\right\rangle$ нетривиально.

ДокаЗАТЕльство. Допустим, что это неверно, т.е.

$$
0 \in\left\langle a \cup S_{1}, a \cup S_{2}, a \cup S_{3}, a \cup S_{4}\right\rangle
$$

и $A=(X(i, j)), 1 \leqslant i \leqslant j \leqslant 4,-$ соответствуюшая определяюшая система. Представим элементы определяюшей системы в минимальной модели $\mathscr{M}$ пространства $\widetilde{V}$ как полиномы по степеням образующей $x$. Имеем

$$
\begin{aligned}
& X(i, i)=x \wedge R_{i}, \quad \text { где }\left[R_{i}\right]=S_{i}, \quad 1 \leqslant i \leqslant 4, \\
& X(i, j)=\sum_{l=0}^{K(i, j)} x^{l} \wedge X_{l}(i, j), \quad 1 \leqslant j-i \leqslant 2 .
\end{aligned}
$$

Заметим, что условие теоремы гарантирует, что

$$
\operatorname{sdeg} X(i, j)<2 m-1<2 \operatorname{codim} Y,
$$

следовательно, образуюшая $y \in \mathscr{M}$ не входит в разложения $(39)$, т.е. $X_{l}(i, j) \in \mathscr{M}_{Y}$ для всех $i, j, l$.

Далее, для определяюшей системы $A$ получаем систему равенств

$$
\begin{cases}\text { 1) } d X_{l}(i, i+1)=\bar{R}_{i} \wedge R_{i+1}, & l=2, i=1,2,3, \\ \text { 2) } d X_{l}(i, i+1)=0, & l \neq 2, i=1,2,3, \\ \text { 3) } d X_{l+1}(i, i+2)=\bar{R}_{i} \wedge X_{l}(i+1, i+2)+\overline{X_{l}(i, i+1)} \wedge R_{i+2}, & l \geqslant 0, i=1,2, \\ 4) d X_{0}(i, i+2)=0, & i=1,2 .\end{cases}
$$


Отсюда вытекает, что $A_{1}\left(X_{j-i+1}(i, j)\right), 1 \leqslant i \leqslant j \leqslant 4$, будет некоторой определяющей системой произведения $\left\langle S_{1}, S_{2}, S_{3}, S_{4}\right\rangle$.

Наконец, для цикла определяющей системы будем иметь

$$
\begin{aligned}
c(A)= & \overline{X(1,1)} \wedge X(2,4)+\overline{X(1,2)} \wedge X(3,4)+\overline{X(1,3)} \wedge X(4,4) \\
= & \sum_{l=0}^{3} x^{l} \wedge P_{l}+x^{4} \wedge\left\{\overline{X_{1}(1,1)} \wedge X_{3}(2,4)+\overline{X_{2}(1,2)} \wedge X_{2}(3,4)\right. \\
& +\overline{X_{3}(1,3)} \wedge X_{1}(4,4)+\overline{X_{0}(1,2)} \wedge X_{4}(3,4)+\overline{X_{1}(1,2)} \wedge X_{3}(3,4) \\
& \left.+\overline{X_{3}(1,2)} \wedge X_{1}(3,4)+\overline{X_{4}(1,2)} \wedge X_{0}(3,4)\right\}+\sum_{l \geqslant 5} x^{l} \wedge P_{l} \\
= & \sum_{l \neq 4} x^{l} \wedge P_{l}+x^{4} \wedge\left\{c\left(A_{1}\right)+\overline{X_{0}(1,2)} \wedge X_{4}(3,4)\right. \\
& \left.+\overline{X_{1}(1,2)} \wedge X_{3}(3,4)+\overline{X_{3}(1,2)} \wedge X_{1}(3,4)+\overline{X_{4}(1,2)} \wedge X_{0}(3,4)\right\},
\end{aligned}
$$

где $P_{l}, l \neq 4,-$ некоторые коциклы, принадлежащие $\mathscr{M}_{Y}$. Заметим, что все $P_{l}$ можно выразить через $X_{r}(i, j)$, но конкретньй вид этих элементов при $l \neq 4$ не имеет значения.

Поскольку $\operatorname{sdeg} c(A)<2 m$, то $c(A)$ может быть точным тогда и только тогда, когда точны все $P_{l}, l=0,1, \ldots$ В частности,

$$
\begin{aligned}
P_{4}=c\left(A_{1}\right)+ & \overline{X_{0}(1,2)} \wedge X_{4}(3,4)+\overline{X_{1}(1,2)} \wedge X_{3}(3,4) \\
& +\overline{X_{3}(1,2)} \wedge X_{1}(3,4)+\overline{X_{4}(1,2)} \wedge X_{0}(3,4)=d Q, \quad Q \in \mathscr{M}_{Y} .
\end{aligned}
$$

Из равенства 2) системы (40) мы получаем, что все $X_{l}(i, i+1), l \neq 2, i=1,2,3$, являются коциклами. Переходя к классам когомологий, получаем

$$
\begin{aligned}
& {\left[c\left(A_{1}\right)\right]=-\left(\left[\overline{X_{0}(1,2)}\right] \cup\left[X_{4}(3,4)\right]+\left[\overline{X_{1}(1,2)}\right] \cup\left[X_{3}(3,4)\right]\right.} \\
& \left.+\left[\overline{X_{3}(1,2)}\right] \cup\left[X_{1}(3,4)\right]+\left[\overline{X_{4}(1,2)}\right] \cup\left[X_{0}(3,4)\right]\right) .
\end{aligned}
$$

Таким образом, $\left[c\left(A_{1}\right)\right] \in N\left(H^{+}(Y) \cdot H^{+}(Y)\right)$, что противоречит сильной нередуцируемости $\left\langle S_{1}, S_{2}, S_{3}, S_{4}\right\rangle$. Лемма и вместе с ней теорема D доказаны.

ЗАМЕчАниЕ. Матричные классы когомологий $\left[X_{l}(1,2)\right],\left[X_{l}(3,4)\right], l=0,1,3,4$, не являются совершенно произвольньпи. Из равенств (40) вытекают необходимые соотношения

$$
\begin{aligned}
& \bar{S}_{1} \cup\left[X_{l}(2,3)\right]+\left[\overline{X_{l}(1,2)}\right] \cup S_{3}=0, \\
& \bar{S}_{2} \cup\left[X_{l}(3,4)\right]+\left[\overline{X_{l}(2,3)}\right] \cup S_{4}=0,
\end{aligned}
$$

При этом матричные классы $\left[X_{l}(2,3)\right], l=0,1,3,4$, обладают только естественньпи ограничениями на степени своих матричных элементов. 


\section{Список литературы}

1. Бабенко И. К., Тайманов И. А. О существовании неформалњных односвязных симплектических многообразий // УМН. 1998. Т. 53. № 5. С. 225-226.

2. Babenko I., Taimanov I. On nonformal simply connected symplectic manifolds // Preprint №358. SFB 288, 1998.

3. McDuff D., Salamon D. Introduction to symplectic topology. Oxford: Clarendon Press, 1995.

4. Thurston $W$. Some simple examples of compact symplectic manifolds // Proc. Amer. Math. Soc. 1976. V. 55. P. 467-468.

5. McDuff D. Examples of symplectic simply-connected manifolds with no Kähler structure // J. Differential Geom. 1984. V. 20. P. 267-277.

6. Gompf R.E. A new construction of symplectic manifolds // Ann. of Math. (2). 1995. V. 142. P. 527-595.

7. Deligne P., Griffiths P., Morgan J., Sullivan D. Real homotopy theory of Kähler manifolds // Invent. Math. 1975. V. 29. P. 245-274.

8. Cordero L. A., Fernandez M., Gray A. Symplectic manifolds with no Kähler structure // Topology. 1986. V. 25. P. 375-380.

9. Tralle A., Oprea J. Symplectic manifolds with no Kähler structure. Berlin: Springer-Verlag, 1997. (Lecture Notes in Math. V. 1661.)

10. Lupton G., Oprea J. Symplectic manifolds and formality // J. Pure Appl. Algebra. 1994. V. 91. P. 193-207.

11. Miller T. On the formality of $k-1$ connected compact manifolds of dimension less than or equal to $4 k-2$ // Illinois J. Math. 1979. V. 23. № 2. P. 253-258.

12. Neisendorfer J., Miller T. Formal and coformal spaces // Illinois J. Math. 1978. V. 22. P. 565-579.

13. Громов М. Дифференциальные соотношения с частными производными. М.: Мир, 1990.

14. Rudyak Y., Tralle A. On Thom spaces, Massey products and non-formal symplectic manifolds // Preprint MPI 1999-71.

15. Sullivan D. Infinitesimal computations in topology // Publ. IHES. 1977. V. 47. P. 269-331.

16. Мальцев А. И. Об одном классе однородных пространств // Изв. АН СССР. 1949. Т. 3. C. 9-32.

17. Nomizu K. On the cohomology of homogeneous spaces of nilpotent Lie groups // Ann. of Math. (2). 1954. V. 59. P. 531-538.

18. Гриффитс Ф., Морган Джс. Рациональная теория гомотопий и дифференциальные формы. М.: Наука, 1990.

19. Stasheff J., Halperin S. Obstructions to homotopy equivalences // Adv. Math. 1979. V. 32. P. 233-279.

20. Kraines D. Massey higher products // Trans. Amer. Math. Soc. 1966. V. 124. P. 431-449.

21. May J. P. Matric Massey products // J. Algebra. 1969. V. 12. P. 533-568.

22. May J.P. The cohomology of augmented algebras and generalized Massey products for $D G A$-algebras // Trans. Amer. Math. Soc. 1966. V. 122. P. 334-340.

23. Smith L. Homological algebra and the Eilenberg-Moore spectral sequence // Trans. Amer. Math. Soc. 1967. V. 129. P. 58-93.

24. Маклейн С. Гомология. М.: Мир, 1966.

25. Moore J. Algébre homologique et homologie des espaces classifiants // Sem. Cartan $(1959 / 60)$, exposé 7 . Ecole Norm. Supér. Paris.

26. Бухштабер В.M. Группы полиномиальных преобразований прямой, неформалњные симплектические многообразия и алгебра Ландвебера-Новикова // УМН. 1999. Т. 54. № 4. C. 161-162.

27. Tischler D. Closed 2-forms and an embedding theorem for symplectic manifolds // J. Differential Geom. 1977. V. 12. P. 229-235.

Московский государственный университет им. М.В. Ломоносова и Университет Монпелье;

Институт математики СО РАН

Поступила в редакцию

E-mail: babenko@nw.math.msu.su, babenko@math.univ-montp2.fr;

taimanov@math.nsc.ru 